\title{
Studies on the Heck Reaction with Alkenyl
}

\section{Phosphates: Can the 1,2-Migration Be Controlled?}

\section{Scope and Limitations}

Jean-Philippe Ebran, Anders L. Hansen, Thomas M. Gøgsig and Troels Skrydstrup*

The Center for Insoluble Protein Structures (inSPIN), Department of Chemistry and the Interdisciplinary Nanoscience Center, University of Aarhus, Langelandsgade 140, 8000 Aarhus, Denmark

Email: ts@chem.au.dk

NMR Data 
Table 2 Entry 7 (Compound 2)

JP573

Archive directory: /export/home/auto/vnmrsys/data

Archive directory:
Sample directory
fille: PROTON

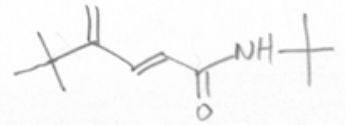

Pulse Sequence: 52 pul

Solvent: $\mathrm{CD} 3 \mathrm{CN}$
Temp $26.0 \mathrm{C} / 299.1 \mathrm{~K}$
Mercury-400B

Re lax. de lay $1.000 \mathrm{sec}$

Pulse 45.0 degrees

Vidth $6410.3 \mathrm{~Hz}$

OBSERVE HII 400,4719454 MHZ

DATA PROCESSI

To 5 ize 65536
Total time 0 min, $50 \mathrm{sec}$

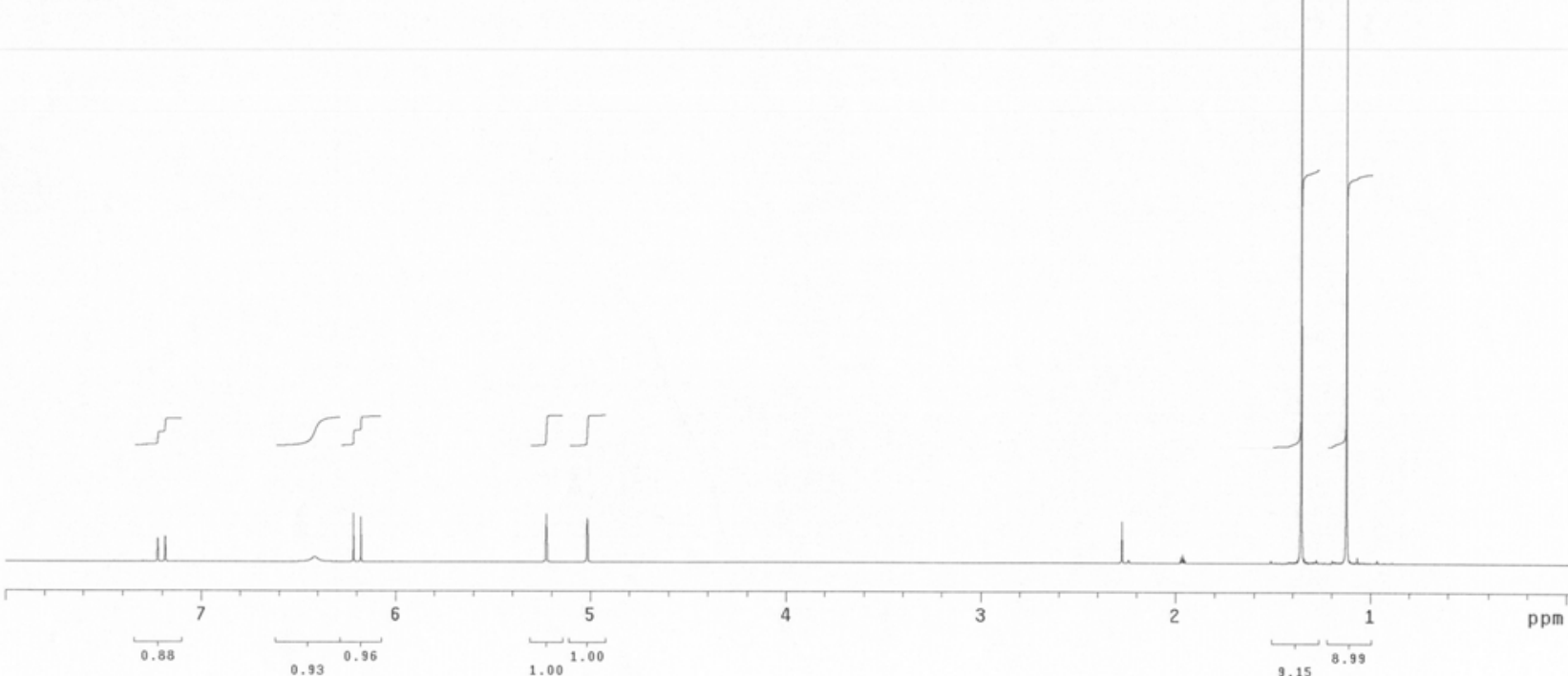

JP573

Archive directory: /export/home/auto/vnmrsys/data

Sample directory:
fille: CARON

Pulse Sequence: 52 pul

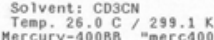

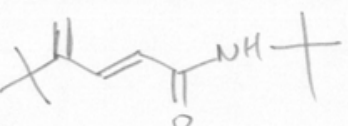

Relax. de lay 1.000 sec

Acq time $1.199 \mathrm{sec}$

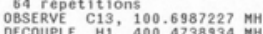

Power $36 \mathrm{~dB}$.

Cont inuous ly on
waL TZ-16 modu
Pated

line broadening $1.0 \mathrm{~Hz}$

Total time 2 min, 26 sec

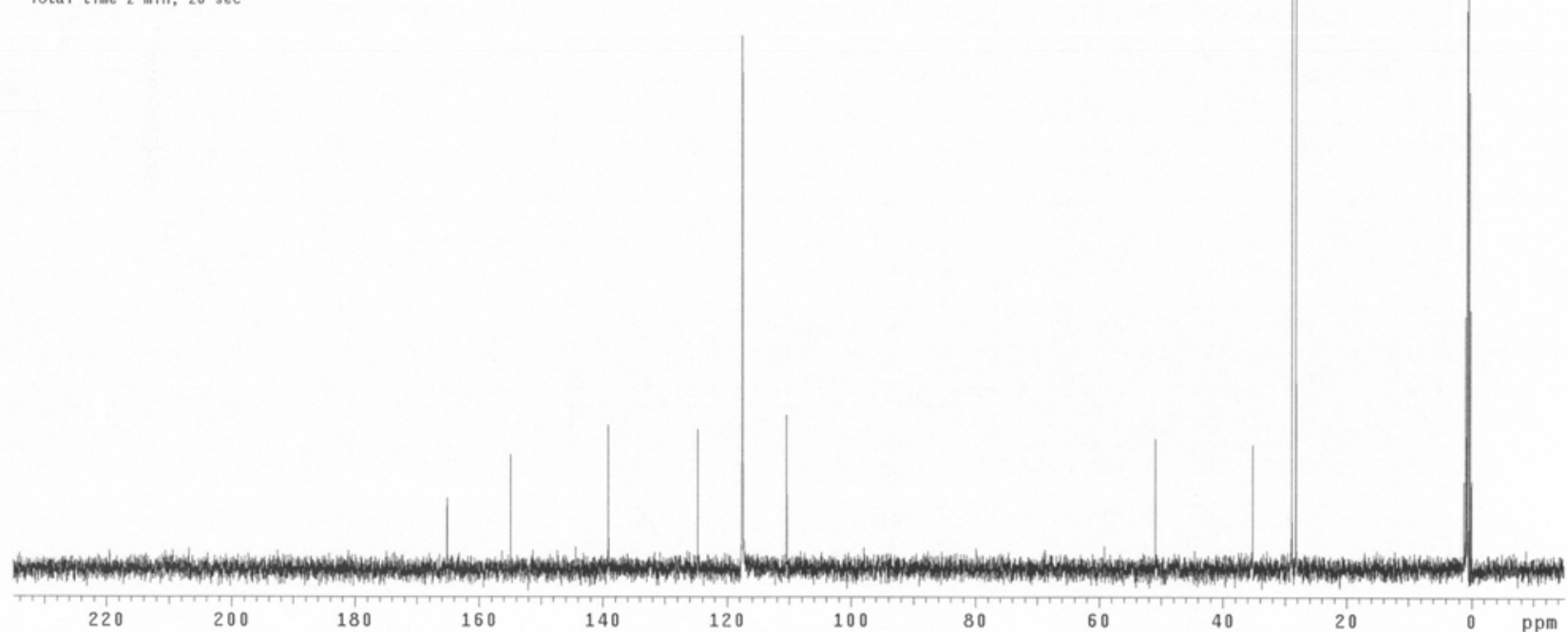


Table 3 Entry $1+2$

JP57

Archive directory: /export/home/auto/vnarsys/data

Sample dir cectory
File: PROTON

Pulse Sequence: 52 pul

Solvent: Co3CN $299.1 \mathrm{~K}$

$\underbrace{\pi}_{0} \mathrm{NH}+1$

Relax. delay 1.000 sec
Pulse 45.0 degrees

Acc time 1.994

32 repetitions
OBSERE H1, 400,4719454 MHE

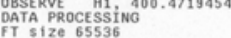

Total time 1 min, $40 \mathrm{sec}$

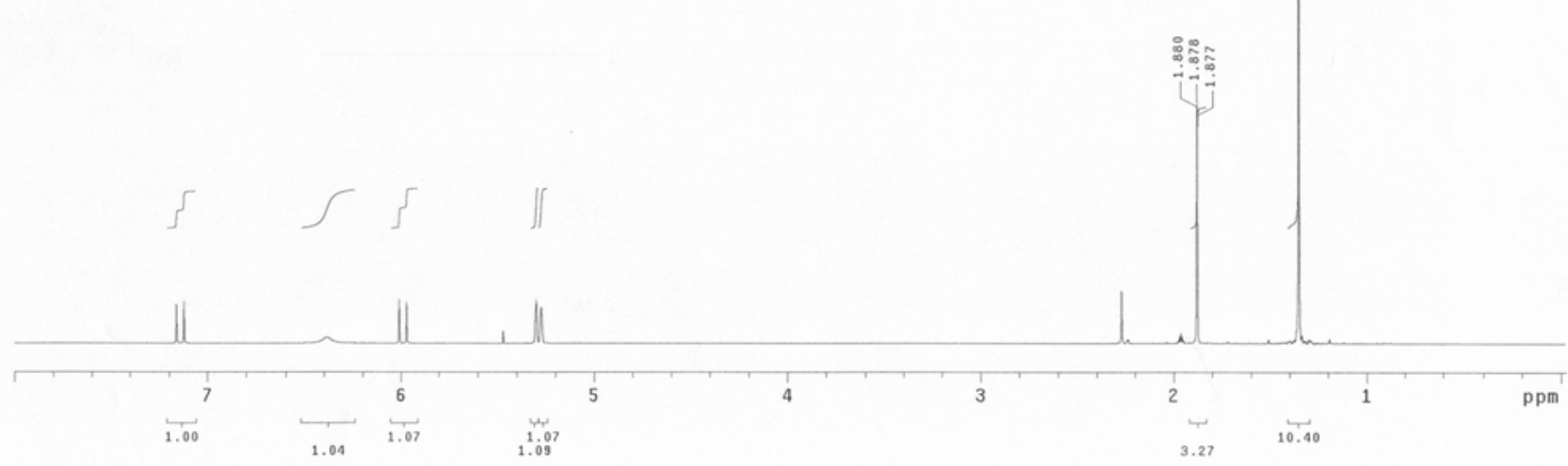

JP575

Archive directory: /export/home/auto/vnmrsys/data

Sample directory
file: CARBON

Pulse Sequence: s2pul

Solvent: CO3CN
Temp. $26.0 \mathrm{C}$ C $299.1 \mathrm{~K}$
Mercury-400B " merc400

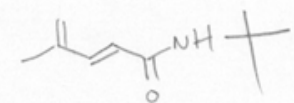

Relax. de lay 1.000 sec

Aca $t$ time $1.199 \mathrm{sec}$

256 repetitions

OSERVE C13, 100.6987212 MHZ
OECOUPLE H1; 400.4738934 HHZ

Power 36 di
continuous on
yatiz-15

WALT2-16 modu lated
OATA PROCESSING
Line broadening $1.0 \mathrm{~Hz}$

Line broadening $1.0 \mathrm{~Hz}$
fT Size 65536
Total

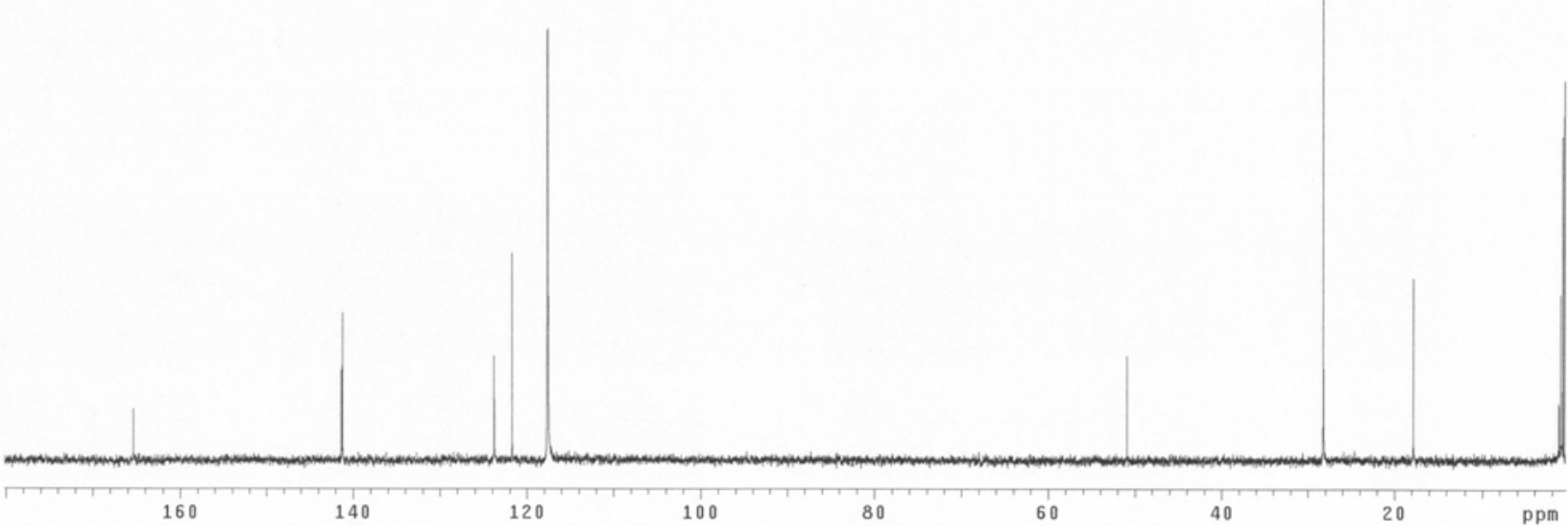


Table 3 Entry 3

JP576-1

Archive directory: /export/home/auto/vnmersys/date

File: PROTON

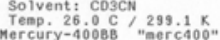

Relax. de lay $1.000 \mathrm{sec}$

Acq. time $1.394 \mathrm{sec}$

Width 6410.3 .

OBSERVE H1, 400.4719454 MHz

FT 512 e 55536
Total time $0 \mathrm{~min}, 50 \mathrm{sec}$
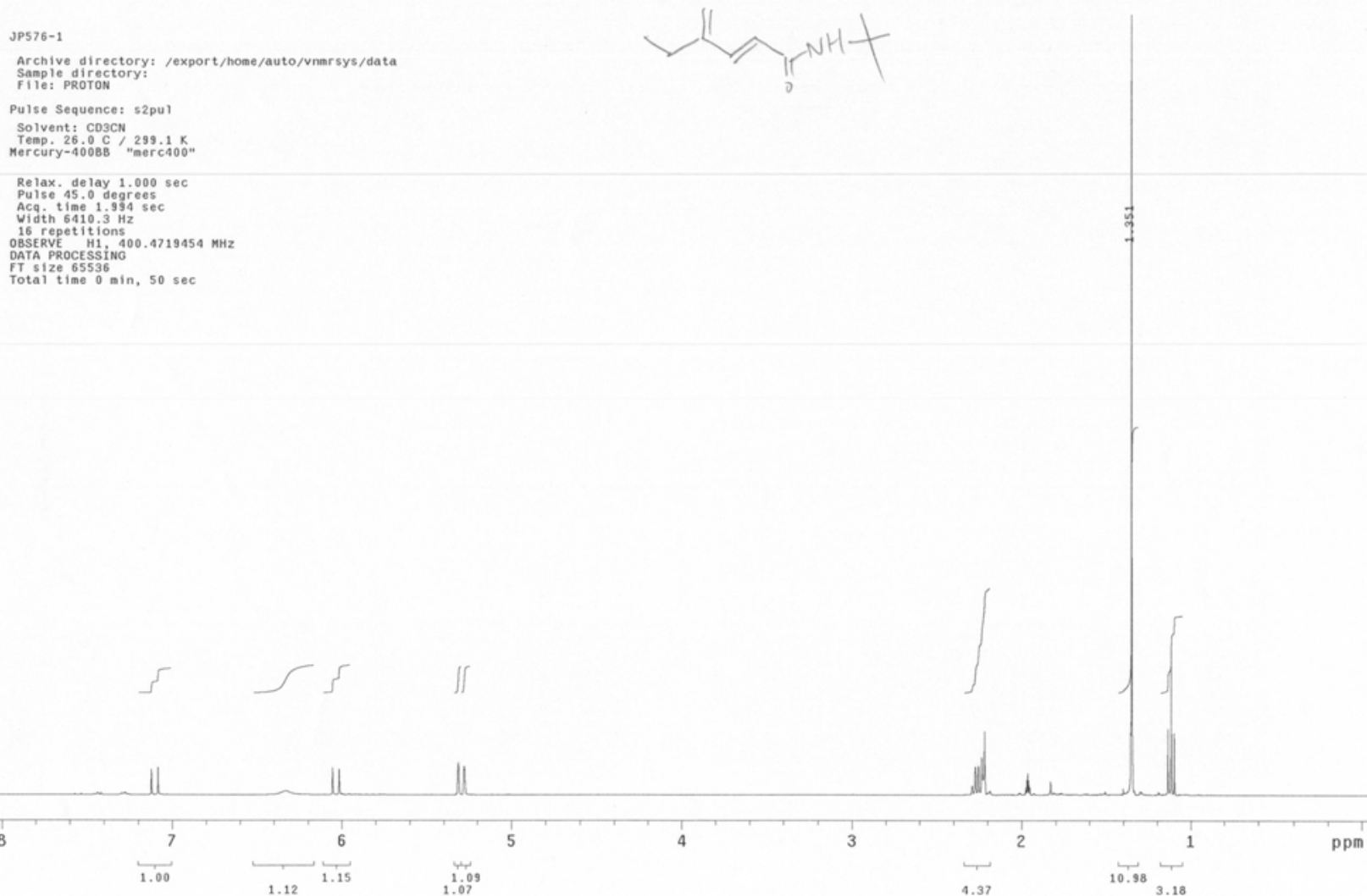

JP576-1

Archive directory: /export/home/auto/vnmrsys/date

Archive directory:
Fille: CARBON

Pulse Sequence: s2pui

Solvent: CD3CN
Temp. $26.0 \mathrm{C}$. $299.1 \mathrm{~K}$
Mercury-400BB "merc400"

Relax. delay 1.000 sec

Acg 2 ime $1.199 \mathrm{sec}$

S12 repetitions
OSEEREE C13, $100.6987204 \mathrm{MHz}$
OECOUPLE H1, $400.4738934 \mathrm{MHZ}$

cont inuous iy on
WaL Tz-16 modulated

OATA PROCESSTNG
IIne broadening $1.0 \mathrm{~Hz}$

FT size 65536.519
Total time $19 \mathrm{~min}, 32 \mathrm{sec}$
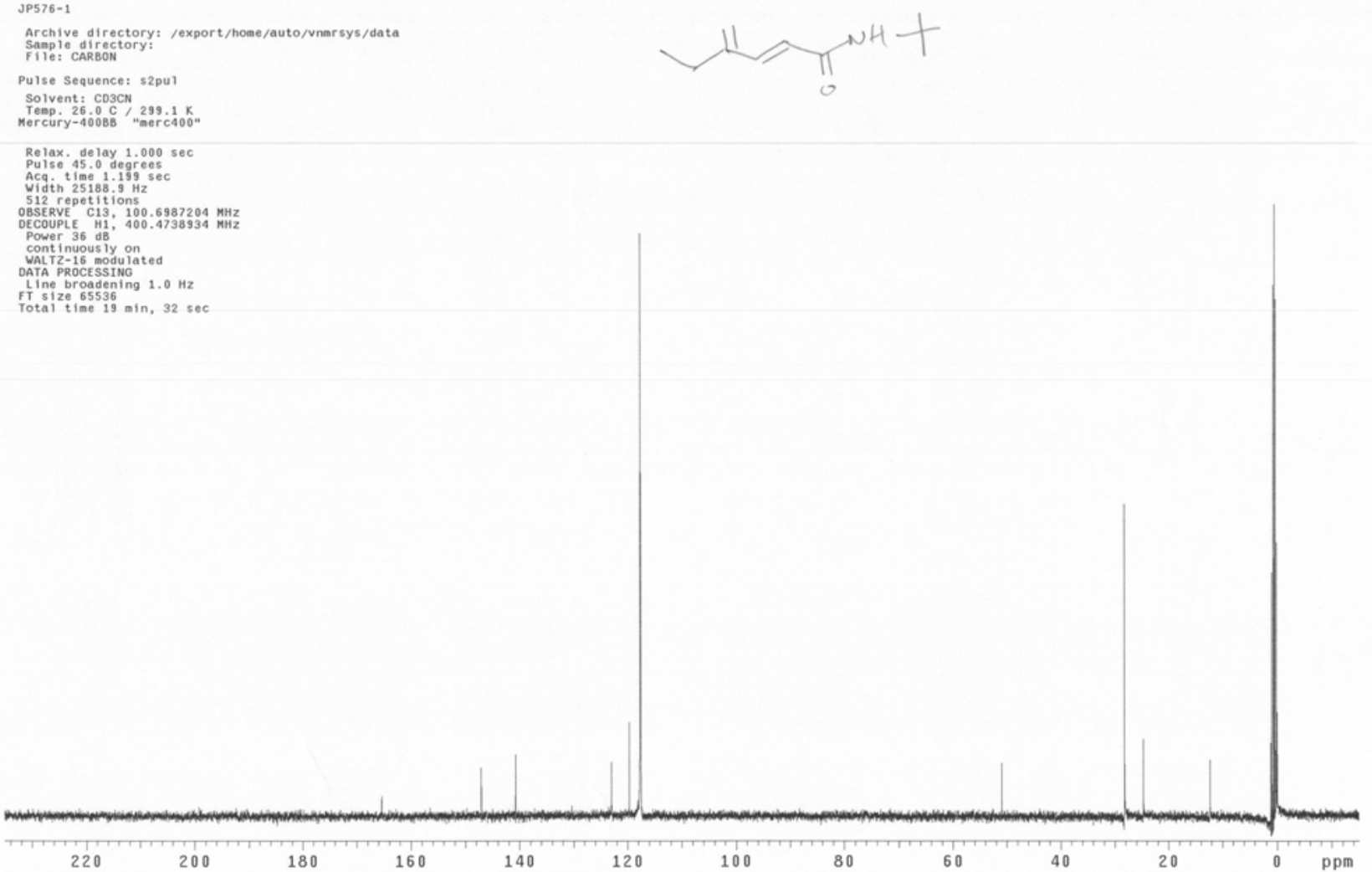
Table 3 Entry 4

JP574

Archive directory: /export/home/auto/ynarsys/data

Sample directory
file: PROTON

Ise sequence: 52 pul

Solvent: CO3CN
Temp. $26.00 \mathrm{C} / 299.1 \mathrm{~K}$
Mercury-4008B

Relax. de lay $1.000 \mathrm{sec}$

Acq. time 1.9954 sec

vidin $6410.3 \mathrm{~Hz}$

OBSERVE H1, 400,4719454 MHZ

Total time 0 min, 50 sec
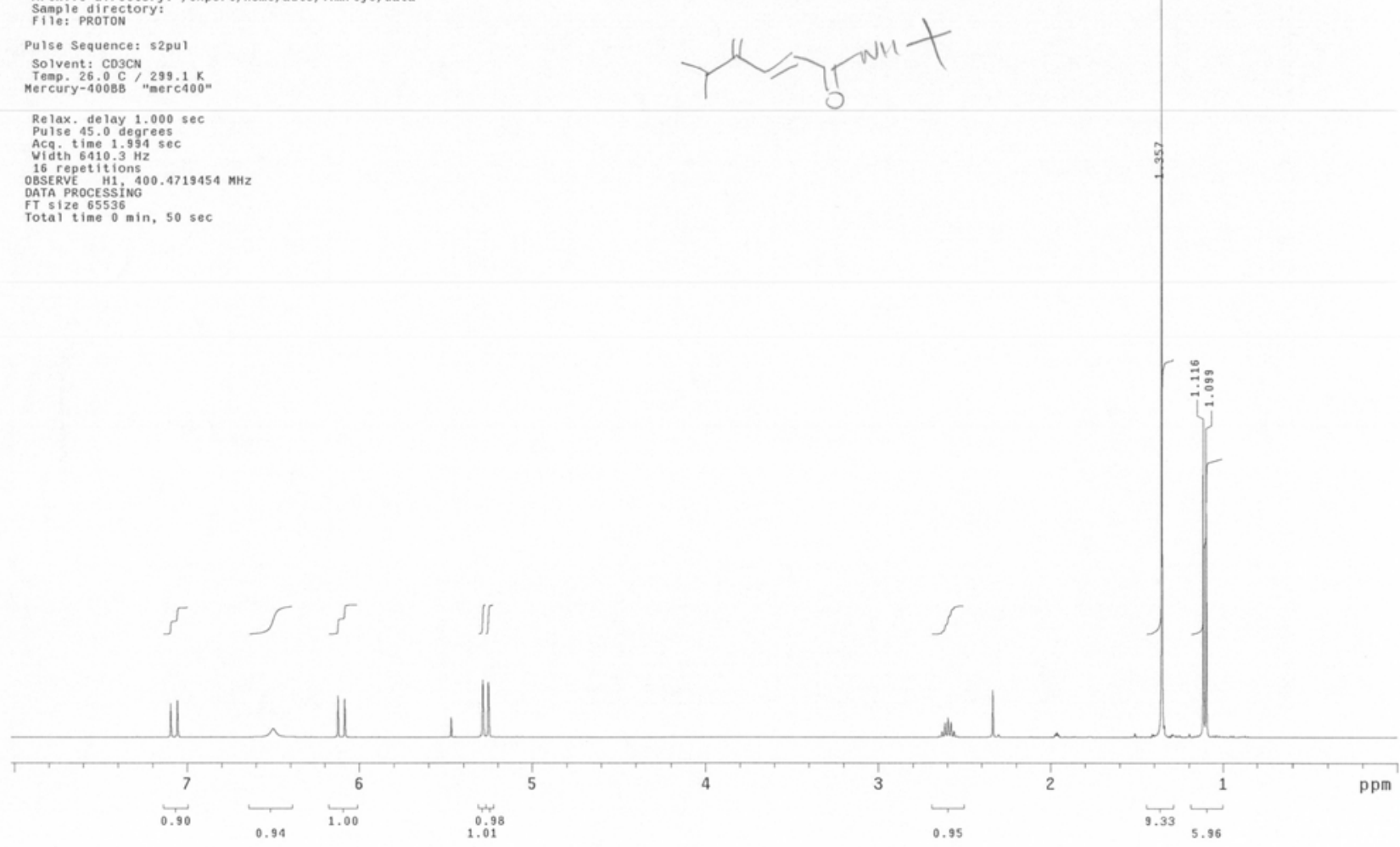

Archive directory: /export/home/auto/vnarsys/dato

ile: CARBON

Pulse Sequence: s2pul

Solvent: Co3CN
Temp. $26.0 \mathrm{C}$ C
Mercury-4008B

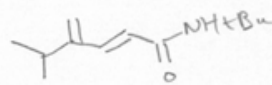

Relax. delay 1,000 sec

Ac time 1 . $199 \mathrm{sec}$

OBSERVE C13, 100.6987235 MH

ECOUPLE H1, 400.4738934 MHI

Cont inuous ly on
Wal $2-16$ modulated

Line broadening $1.0 \mathrm{~Hz}$

fit

. 
Table 3 Entry 5

JP $581-1$

Archive directory: /export/home/auto/vnarsys/data
Sample directory

Sample directory:
Fille: PROTON

Pulse Sequence: s2pul

Solvent: $\mathrm{CD} C \mathrm{CN}$
Temp. $26.0 \mathrm{C} / 29.1 \mathrm{~K}$
Mercury $400 \mathrm{~B}$ " $2996400 "$

Relax. de lay $1.000 \mathrm{sec}$

Pct. $t$ ime $1.994 \mathrm{sec}$

Vidit $6410.3 \mathrm{~Hz}$

OBSERVE H1 400.4719454 MHZ

DATA PRoCESSING
FT SIIZ 65536
Total time 0 .
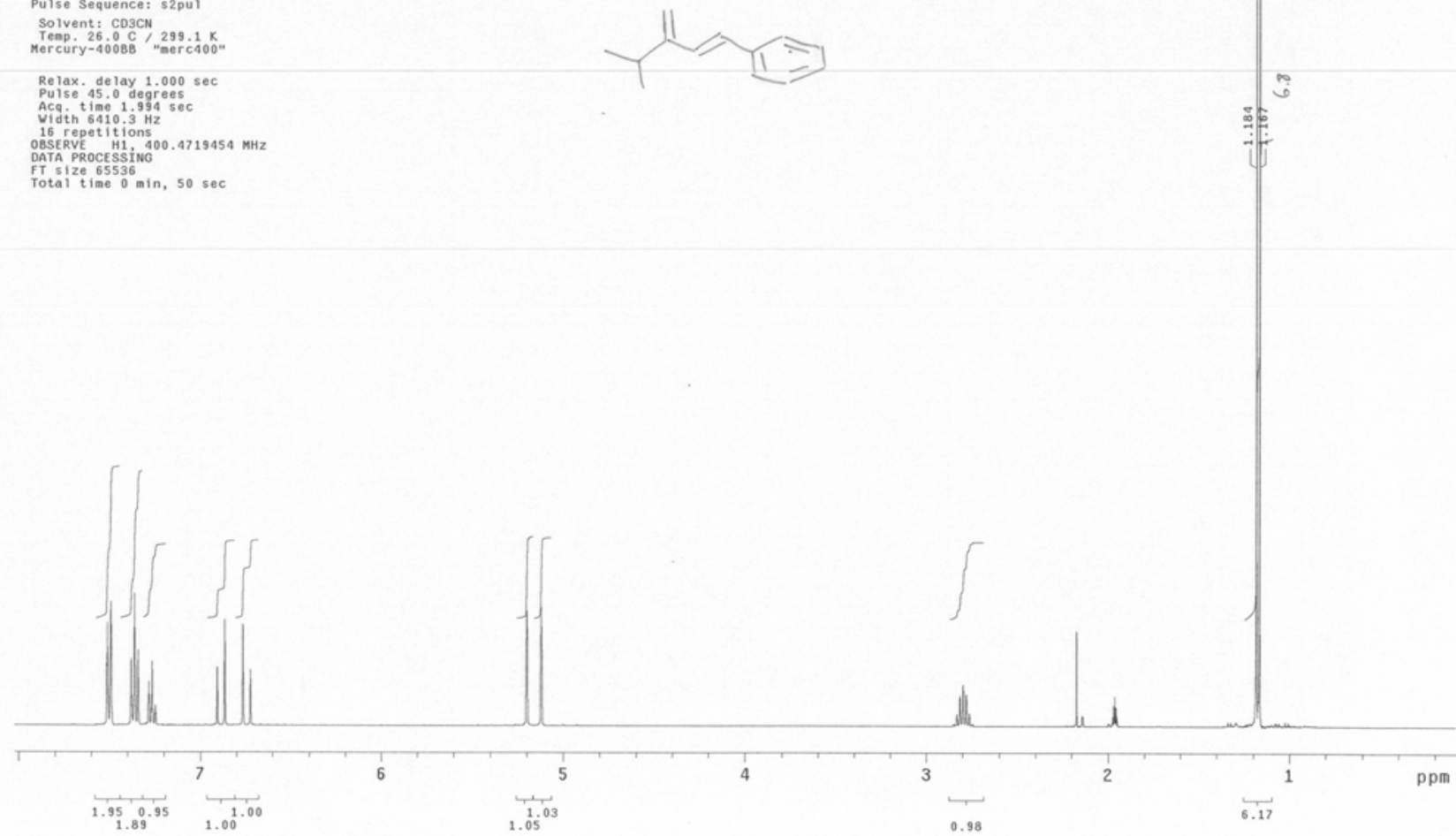

JPSB1-1

Archive directory: /export/home/auto/vnersys/date

Sample directory:
FIIU: CARBON

Pulse Sequence: s2pur

Solvent: CD3CN
Temp. $26.0 \mathrm{C} C \mathrm{C} / 299.1 \mathrm{~K}$
Mercury-400B

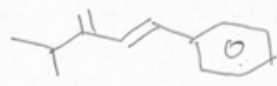

Relax. delay $1.000 \mathrm{sec}$

Acg. time $1.199 \mathrm{sec}$

256 repetitions

OSEREE C13, 100.6987219 MHZ
OECOUPLE H1; $400.4738934 \mathrm{MHZ}$

Pover 36 dB
continuous ly on
walt $T 2-16$ modulat

WALT2-16 modulated
DATA PRoCESSING
Line broadening 1.0 H.

FT
Totaze 655356 ing $1.0 \mathrm{~Hz}$

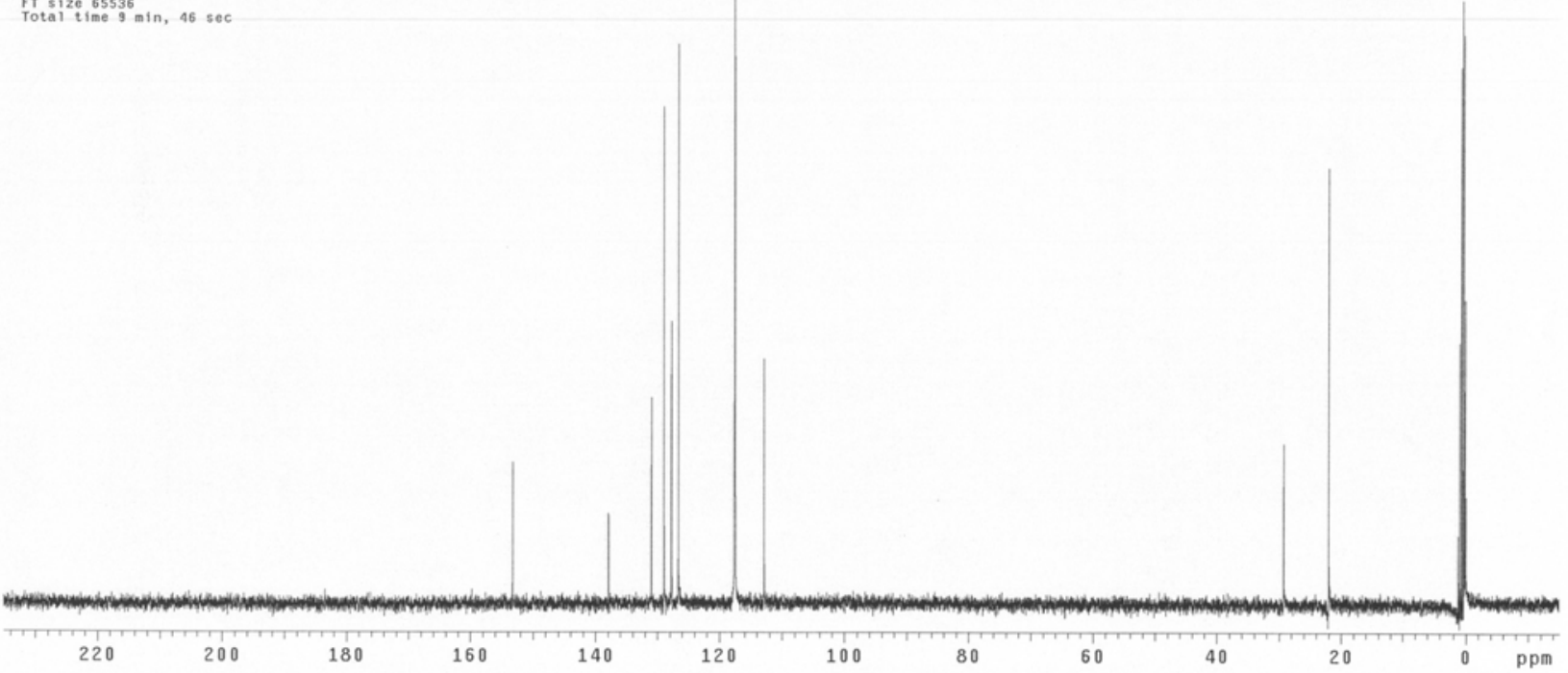


Table 3 Entry 6

sp 577-pur $\mathrm{CD}_{2} \mathrm{Cl}_{2}$

Archive directory: /export/home/auto/vnar sys/data
Sample

Sample directory
Fille: PRoTow

Pulse Sequence: $52 \mathrm{pu}$

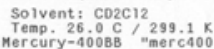

$\mathrm{Ph}_{H} \mathrm{H}_{0} \mathrm{NH}+$

Relax. de loy

Acat time 19394

16 repettions

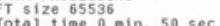
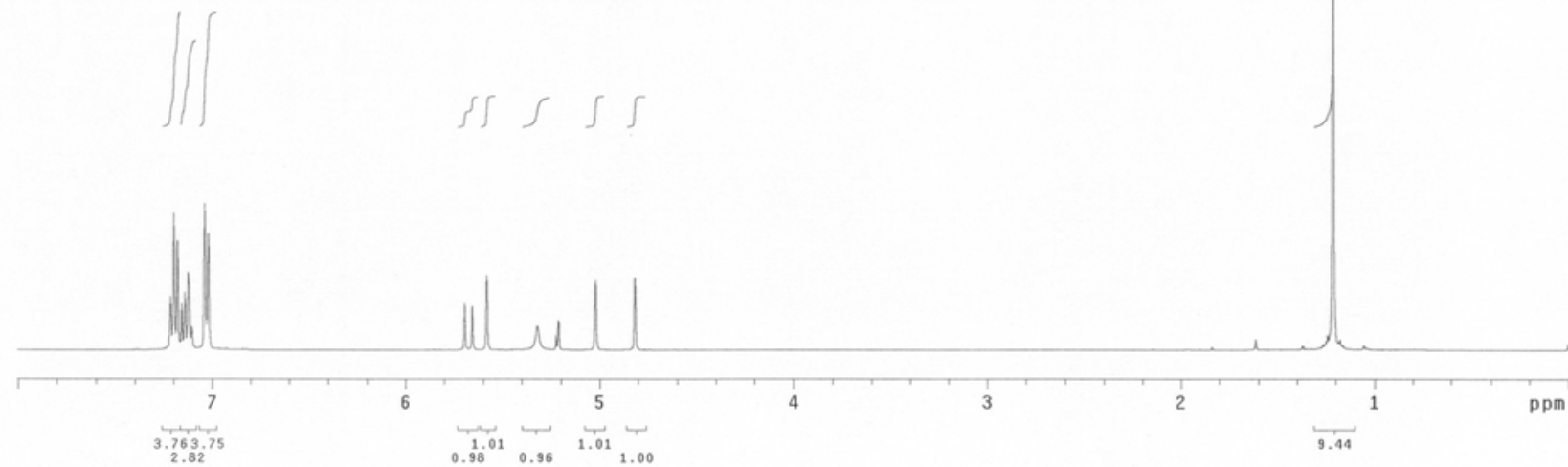

JP 577-pur

Archive directory: /export/home/auto/vnarsys/data

Sample directory:
File: CARBON

Pulse Sequence: s2pul

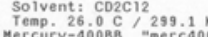

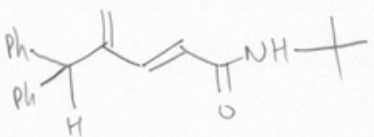

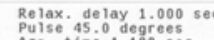

Acq. time 1.199 sec

BSERVE C13, 100.6983874 MHZ

Power $36,400.4725438$ Mit

cont inuous Iy on
val TZ-16 modu lated

Line broadening $1.0 \mathrm{~Hz}$

Tot 512 . 65536 .

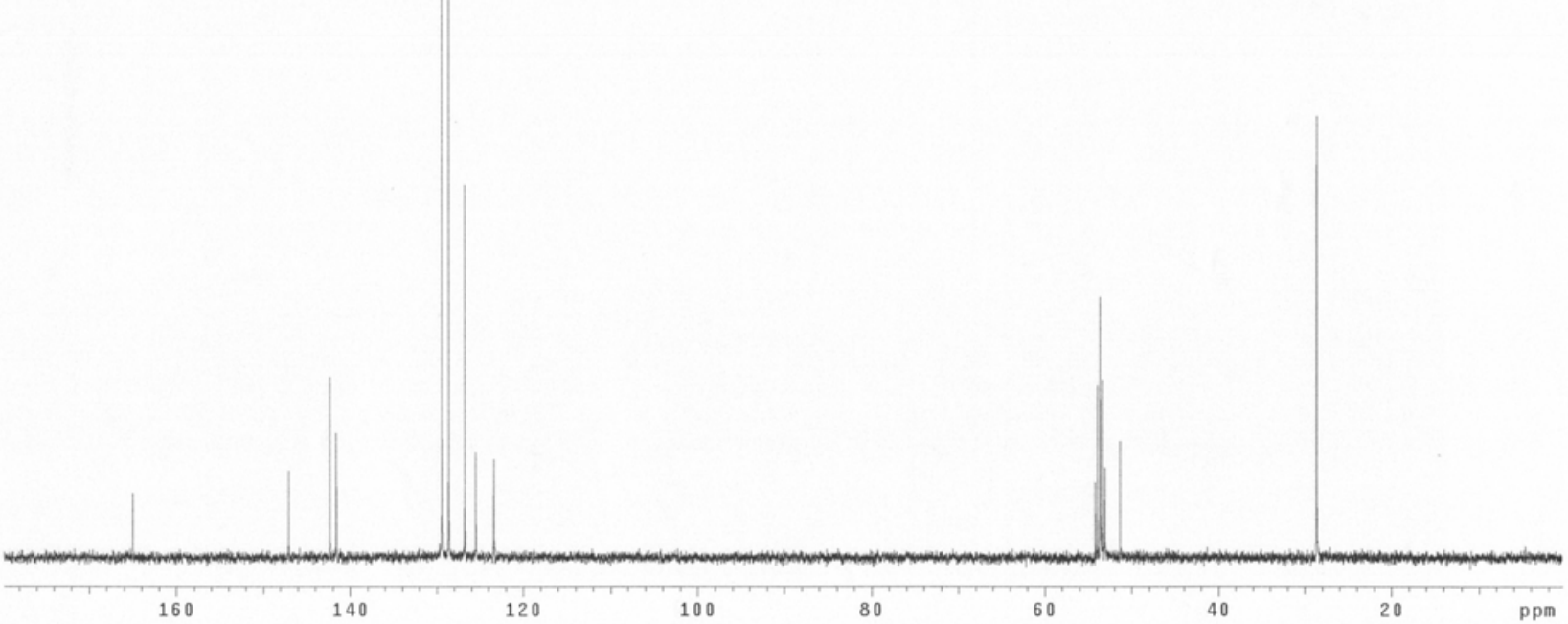


Table 3 Entry 7

JP 589

Archive directory: /export/home/auto/vnimsys/data
Sample directory:

Pulse Sequence: $52 \mathrm{pu}$

Solvent: $\mathrm{CO}_{2} \mathrm{C}_{12} 2 \mathrm{O}$

Mercury-4008 "merc400"

Relax. delay 1.000 sec
Pulse 45.0 degrees

Aca. ${ }^{\text {time }} 1.994$

16 repetitions

DATA PROCESSING

FT 5 ize 65536
Total time 0 min, $50 \mathrm{sec}$

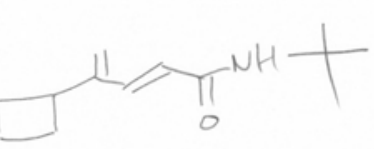

Filename: AuH

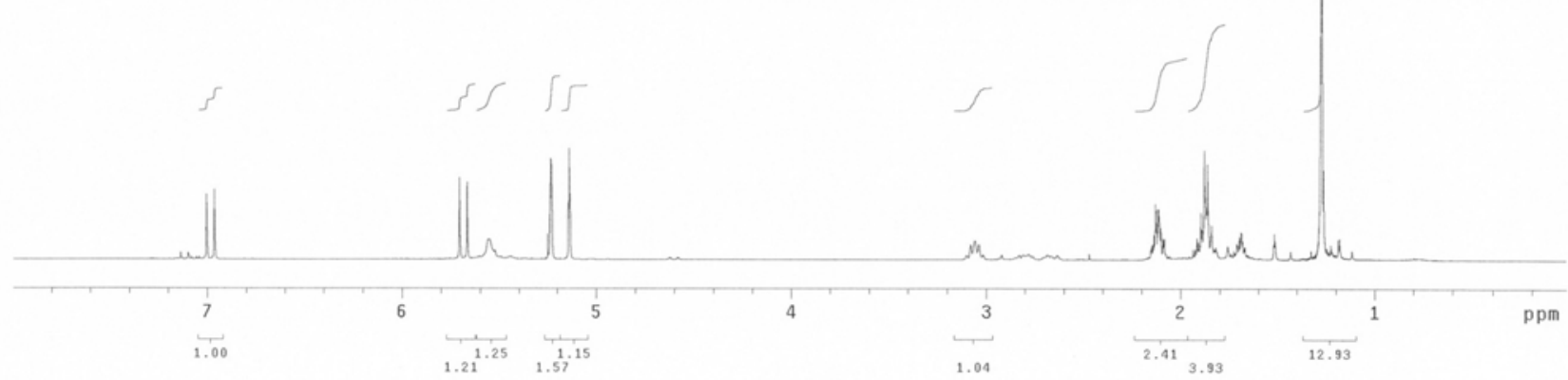

589

Archive directory: /export/home/auto/vnarsys/data

Sample directory
Fille: CARBON

Pulse Sequence: $52 \mathrm{pur}$

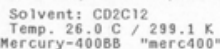

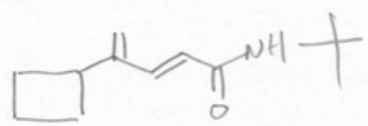

Relax. de lay 1.000 sec

Vidth $25188,9 \mathrm{~Hz}$

DESEVE C13, 100.6983874 MH:
DECOUPLE H1, 400.4725438 MH

Power 36 de de $^{-}$

Cont inuous ly on
val $2-16$ modulate

DATA PROCESSING
Line broadening $1.0 \mathrm{~Hz}$

Fr size 65536 .

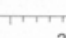

220

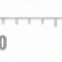

200

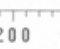

180

160

140

120

100

80

60

40

20

$0 \quad \mathrm{ppm}$ 
Table 3 Entry 8

625

exp1 s2pu1

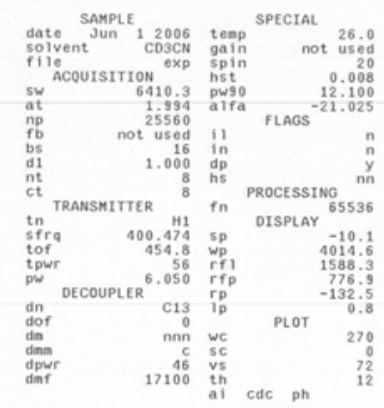<smiles>C=C(C=CC(=C)C1CCCCC1)NC(C)C</smiles>

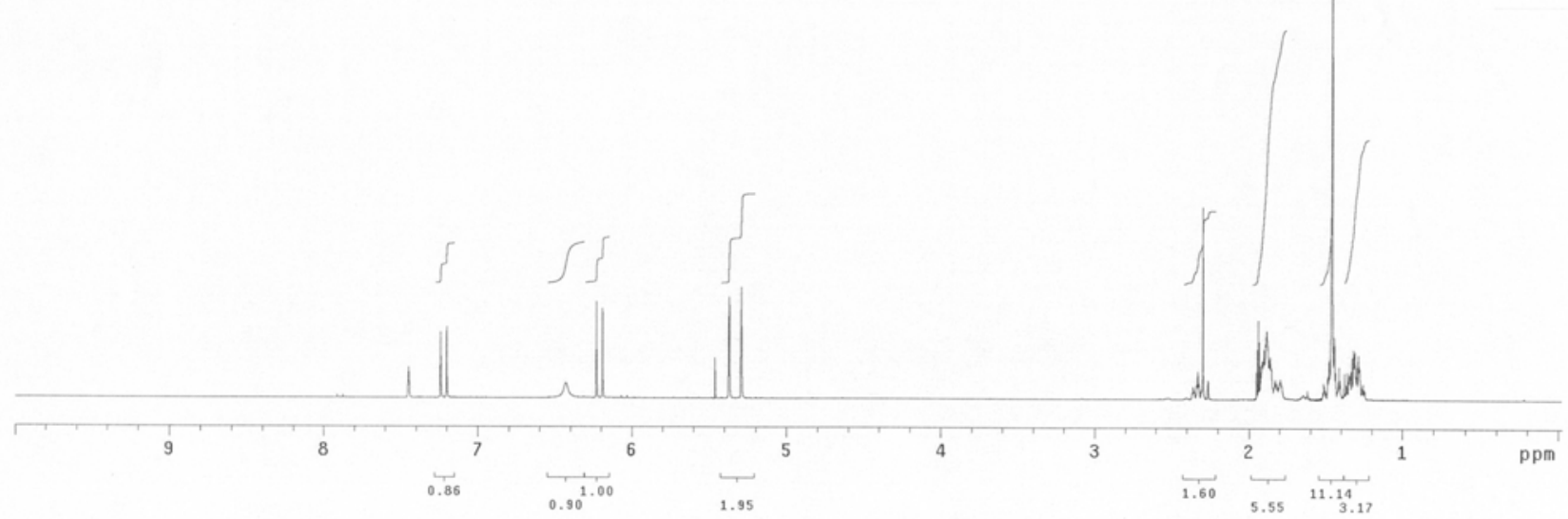

625

exp1 52 pul

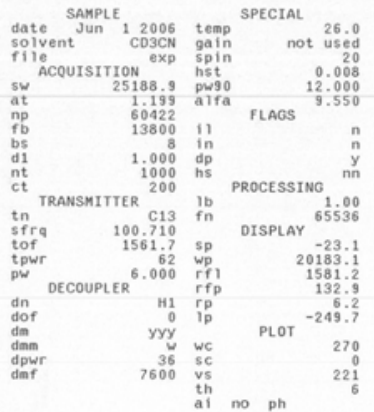

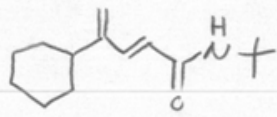

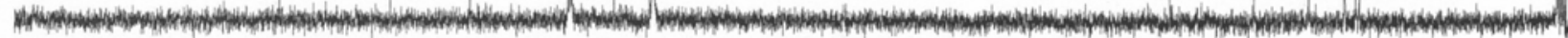

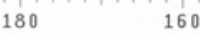

$160 \quad 140$

120

100

80

60 
Table 3 Entry 9

$\operatorname{exp1}$ s2pul

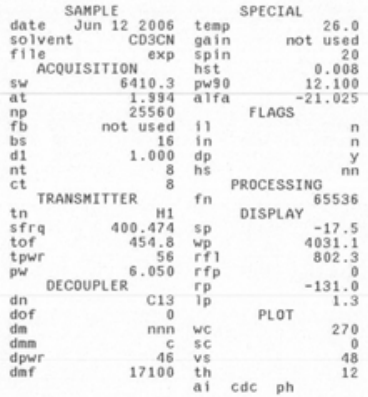<smiles>C=C(/C=C/C(=O)OCCNN=C(C)OC(C)(C)C)C1CCCCC1</smiles>

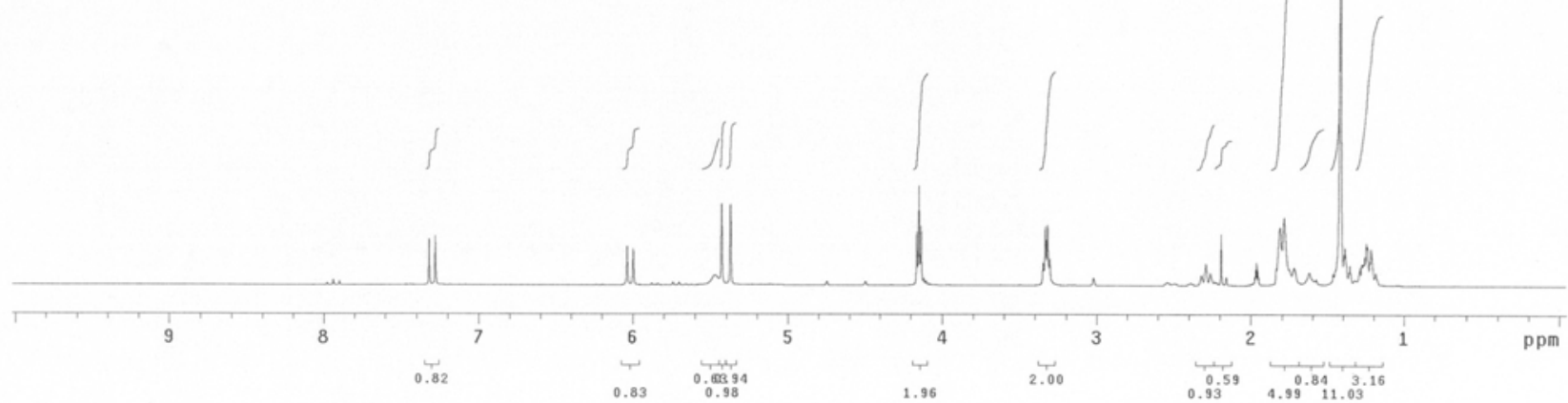

639

exp1 s2pul

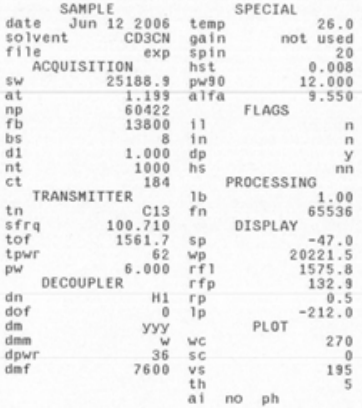<smiles>C=C(/C=C/C(=O)OCCN=CC(=O)OC(C)(C)C)C1CCCCC1</smiles>

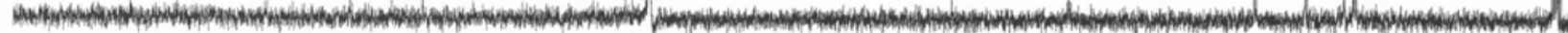

180

160

140

120

100

80

60

40

$20 \quad$ ppm 
Table 3 Entry 10
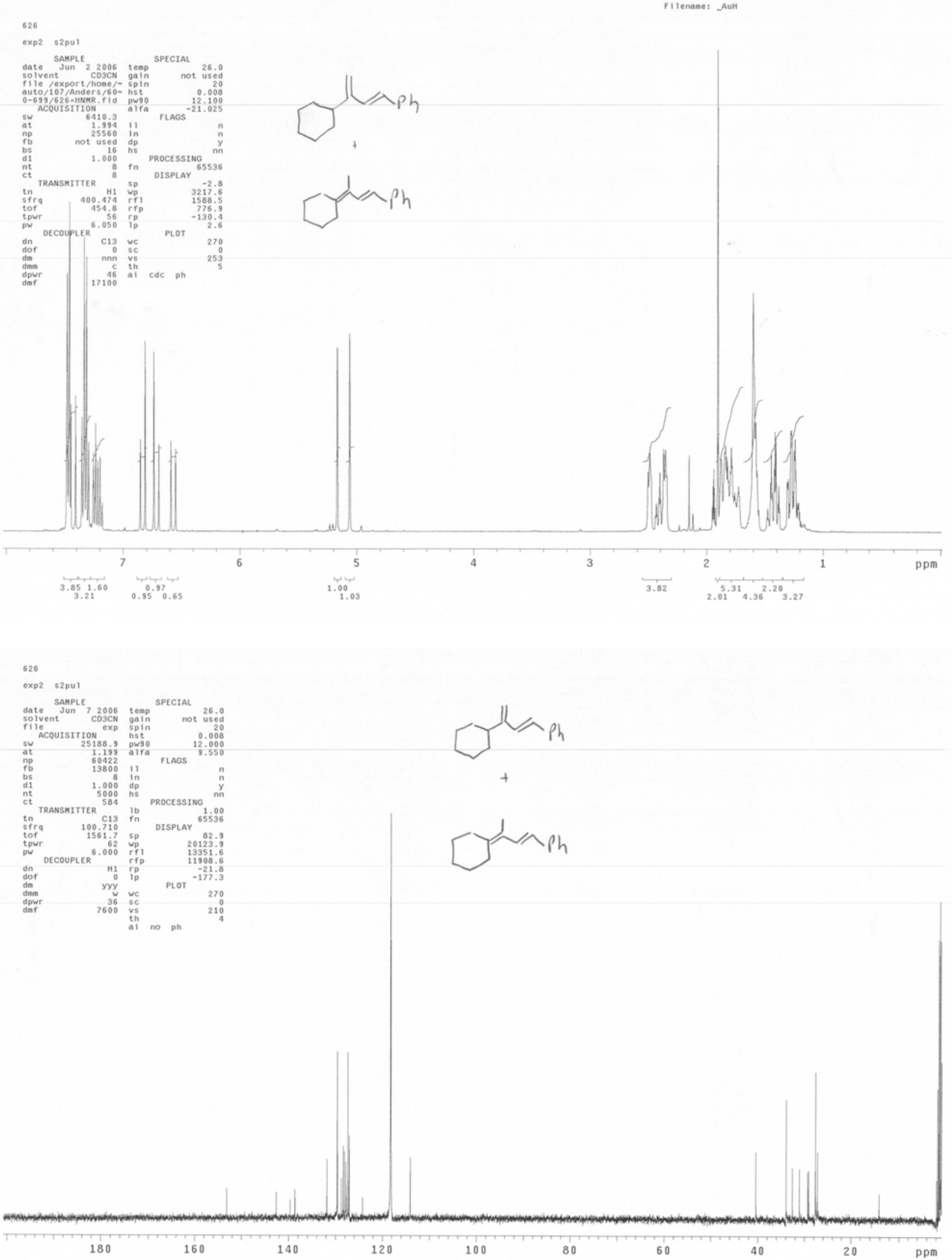
Table 4 Entry 2

JP 578

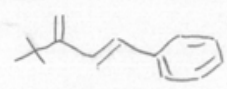

Archive directory: /exporthrome/auto/vnmesys/data

Sample directory
File: PROTON

Pulse Sequence: s2pul

Solvent: CD3CN
Temp. $26.0 \mathrm{C}$ C $299.1 \mathrm{~K}$
Mercury-400BB

Relax. de lay $1.000 \mathrm{sec}$

Puct $t$ ine 1.994 sec

Vidth $6410.3 \mathrm{~Hz}$

OBSERVE H1 400, 4719454 MHZ

DAA PPOCESSING
FT Size 65536 .
Total tine

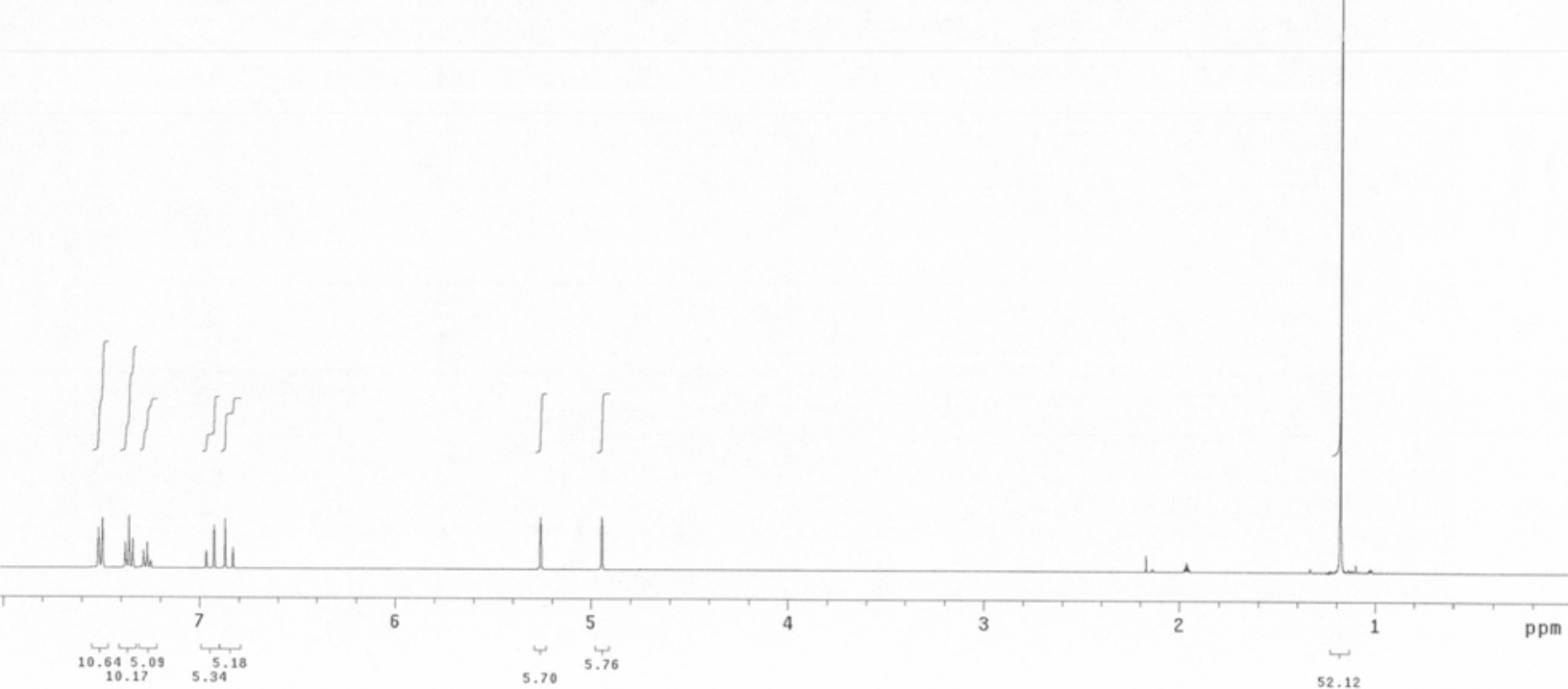

578

Archive directory: /export/home/auto/vnarsys/date

Fille: CARBON

Pulse Sequence: s2put

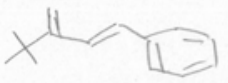

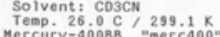

Relax. de lay $1.000 \mathrm{sec}$

Acy 45.0 degrees

Width 25188.9 . Hz

OBSERE C13, 100.6987235 MHZ
OECOUPLE H1, 400.4738934 MHZ

Power 36 ds

WALT TZ-16 modu inated
DATA PROCESSING

Line broadening $1.0 \mathrm{~Hz}$

Totai time 9 in, 46 sec

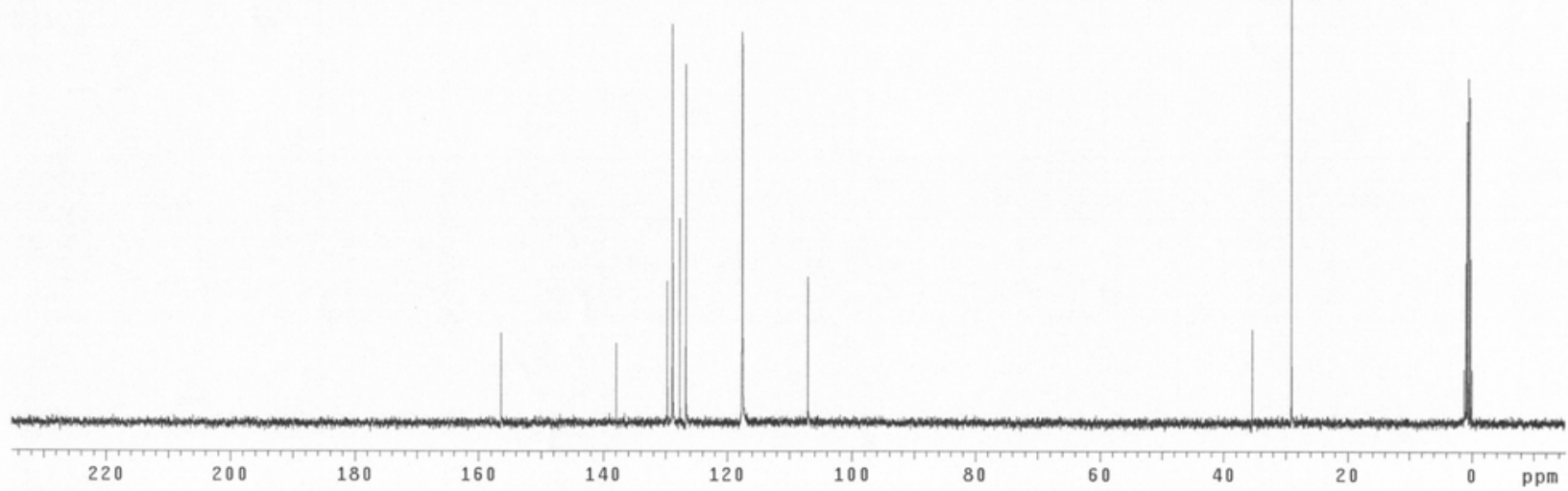


Table 4 Entry 3

611 $1: 1 \mathrm{CD} \mathrm{CN}$ : $\mathrm{C} 6 \mathrm{DS}$

exp2 s2pul

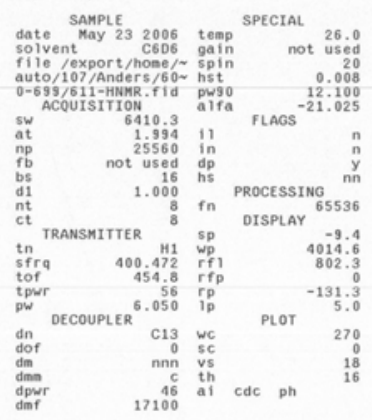
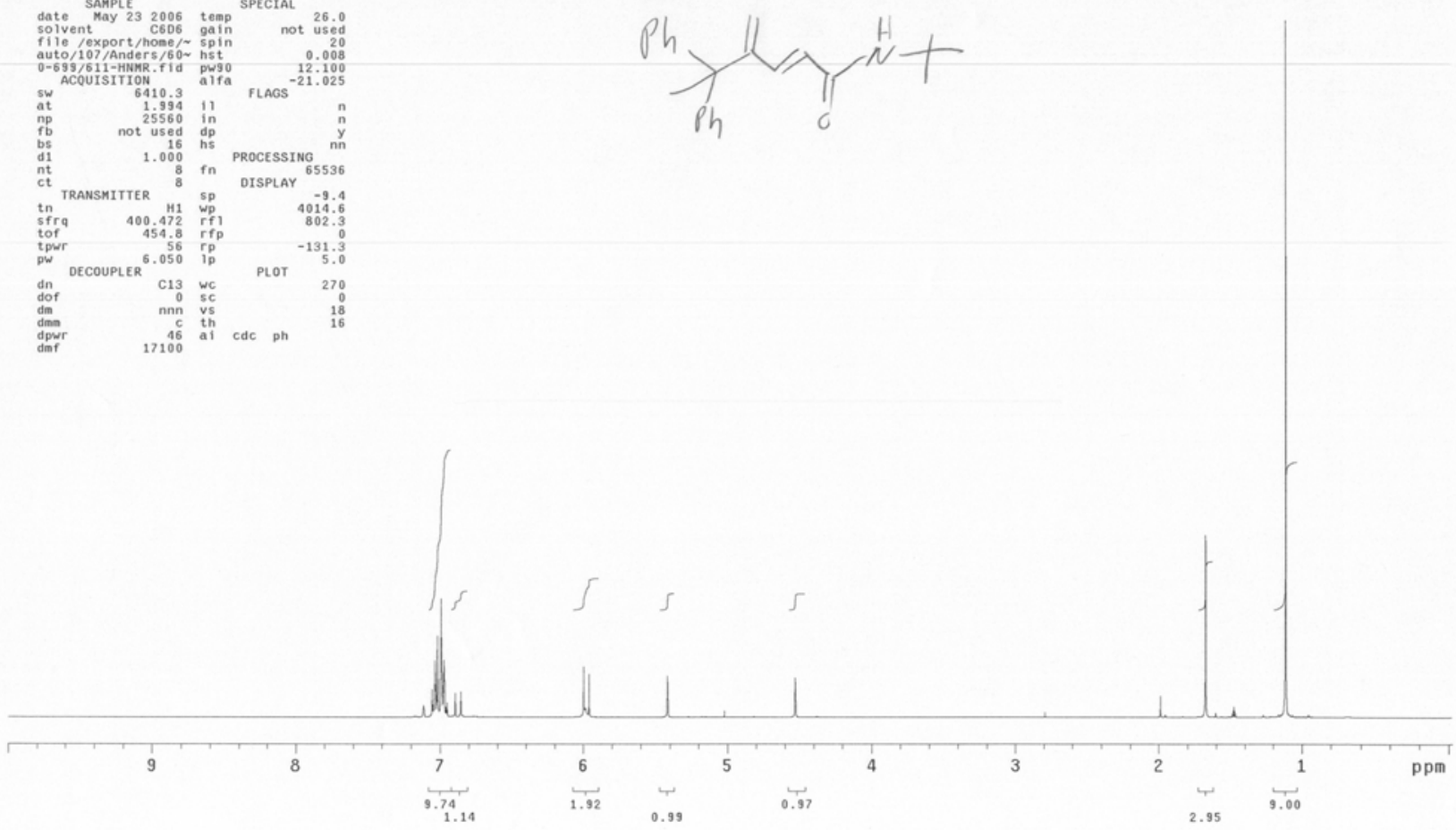

611
$1: 1$ CD3CN : C606

exp2 s2pul

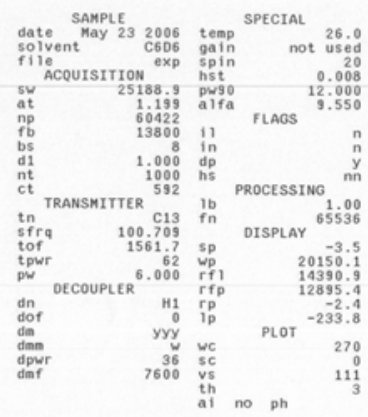<smiles>C=C(C=CC(=C)C(C)(C)C)NC</smiles>

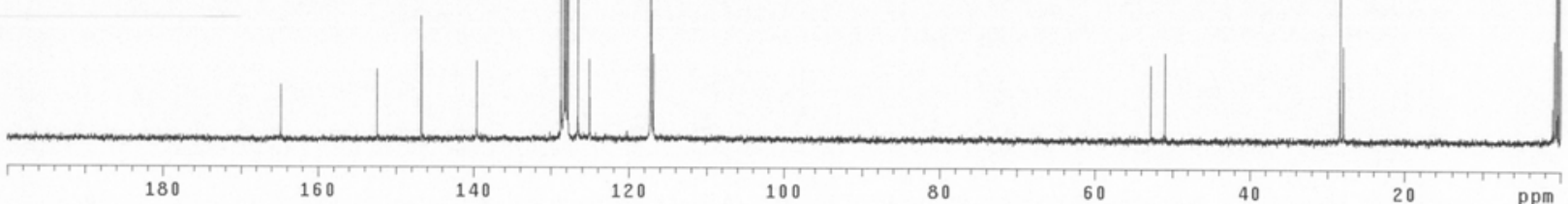


Table 4 Entry 4

JP585

Archive directory: /export/home/auto/vnmrsys/data

Archive directory:
Sample directory:
File: PROTON

Pulse Sequence: s2put

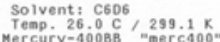

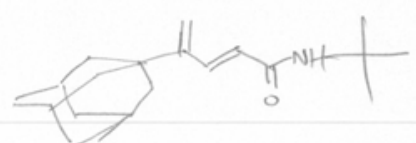

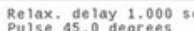

Acge time 1.994 sec

16 repetitions
OBSERVE

DATA PROCESSINC

列
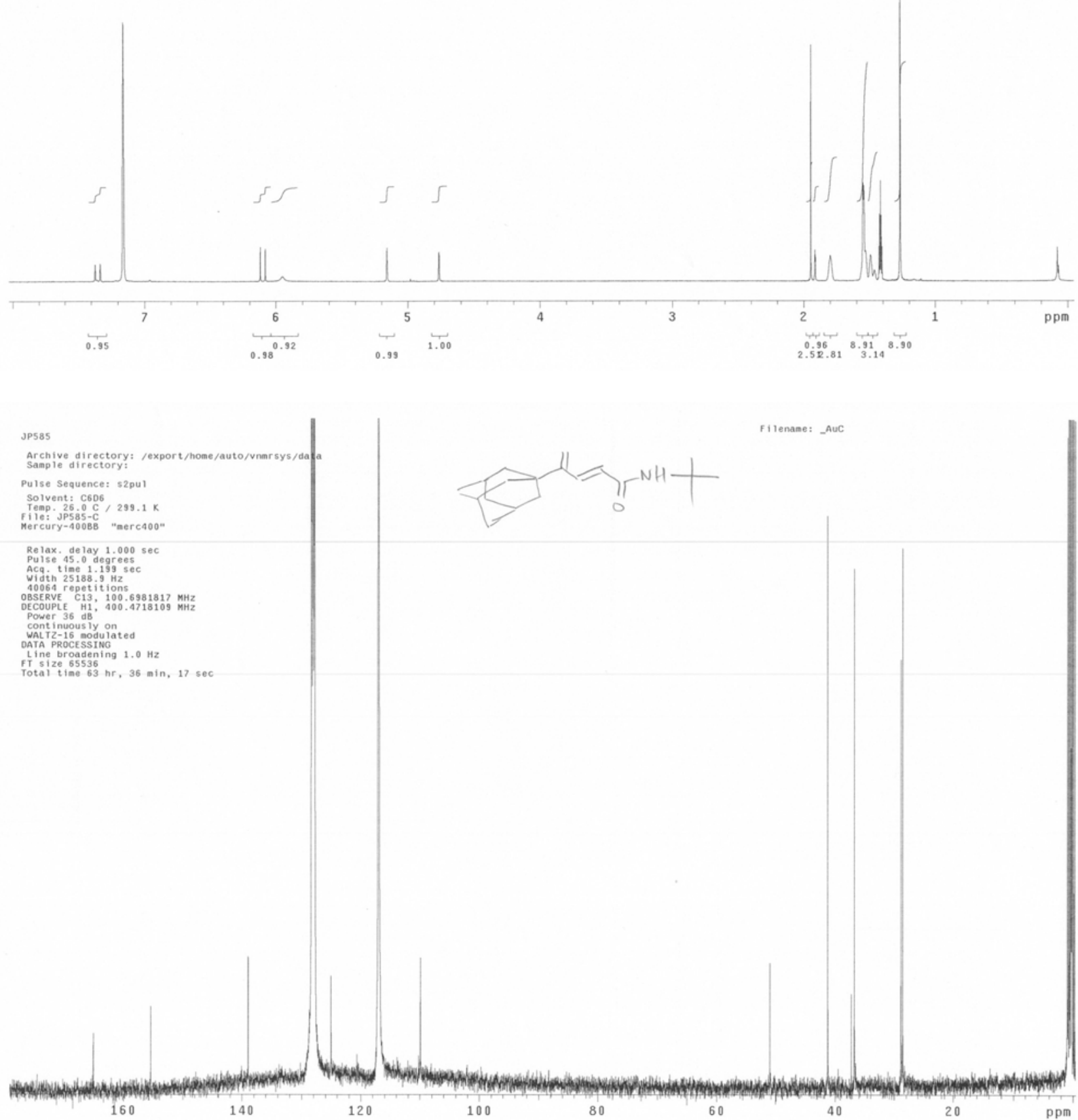
Table 4 Entry 5

627

Sexp1 52 pur
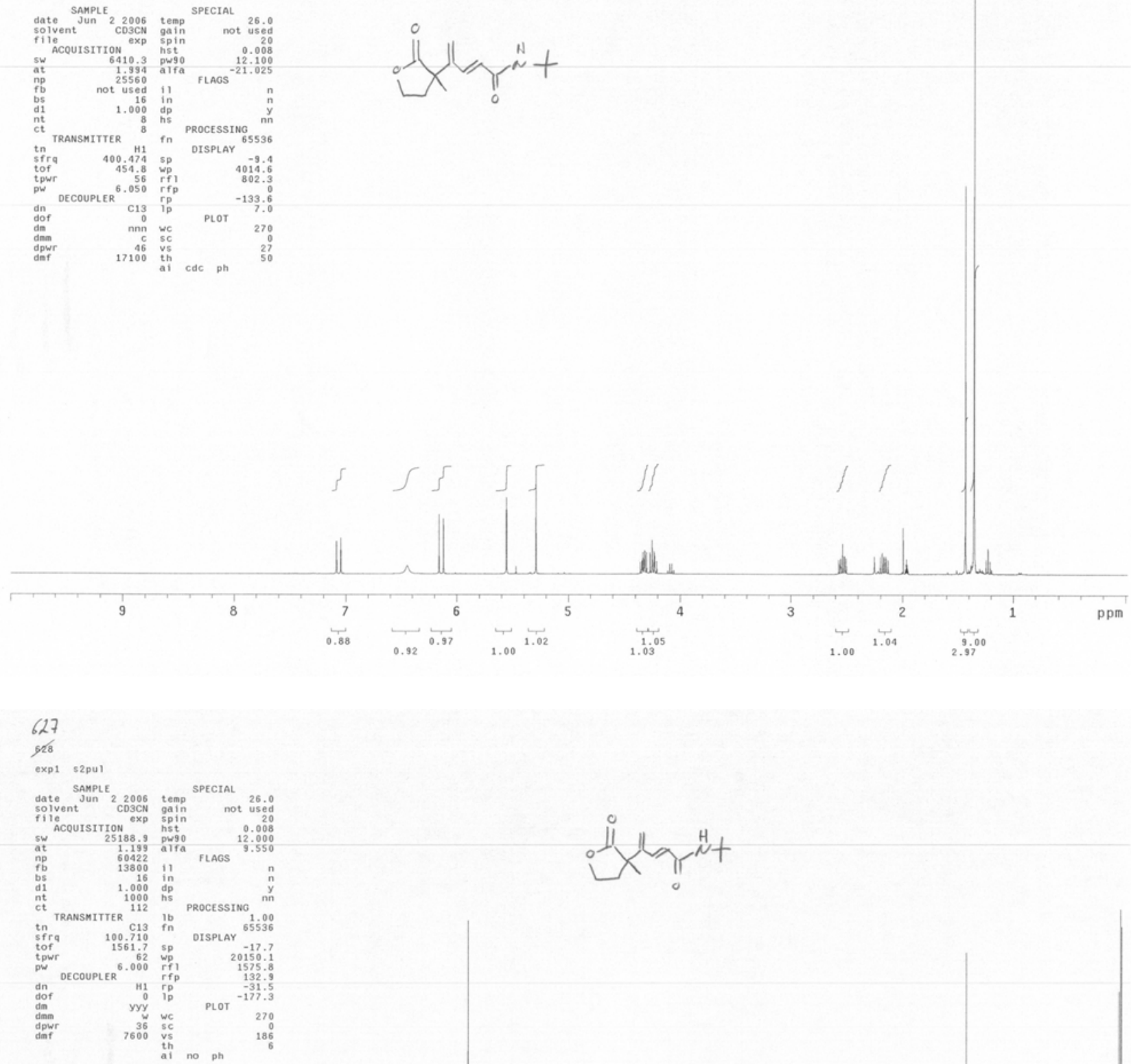

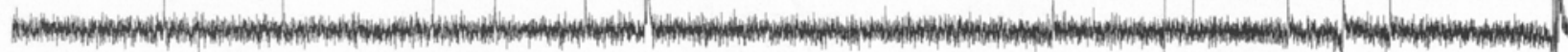

$180 \quad 160$

140

120

100 
Table 4 Entry 6

640

exp1 s2pu1

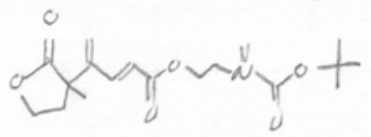

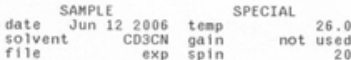

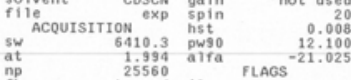

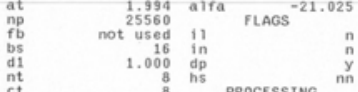

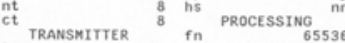

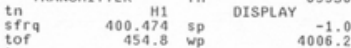

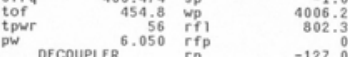

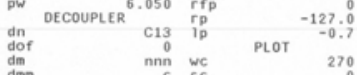

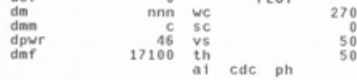

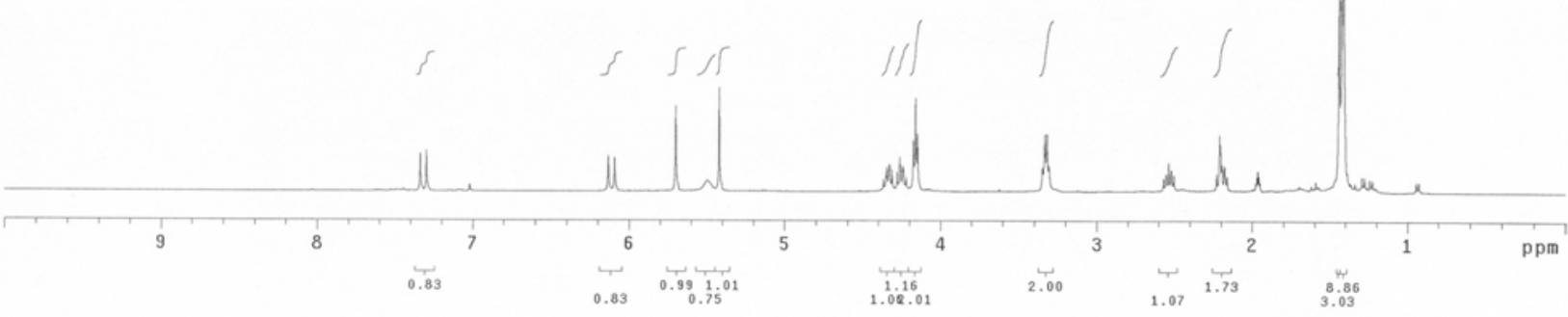

Archive directory: /export/home/auto/vnmrsys/date

Sample directory:
File: CARBON

Pulse Sequence: s2pul

Solvent: CD3CN
Temp. $26.00 \mathrm{CN} / 299.1 \mathrm{~K}$
Mercury-400BB

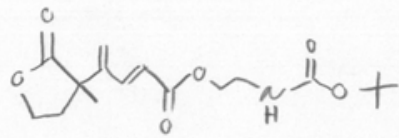

Relax. delay $1.000 \mathrm{sec}$
Pulse 45.0 degrees

Acc itime $1.199 \mathrm{sec}$

OBSERE C13, 100.6986520 MHZ

Power 36 de
cont nuous $1 \mathrm{y}$ on

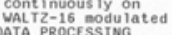

tine broadening $1.0 \mathrm{~Hz}$

Total time 45 min, $6 \mathrm{sec}$

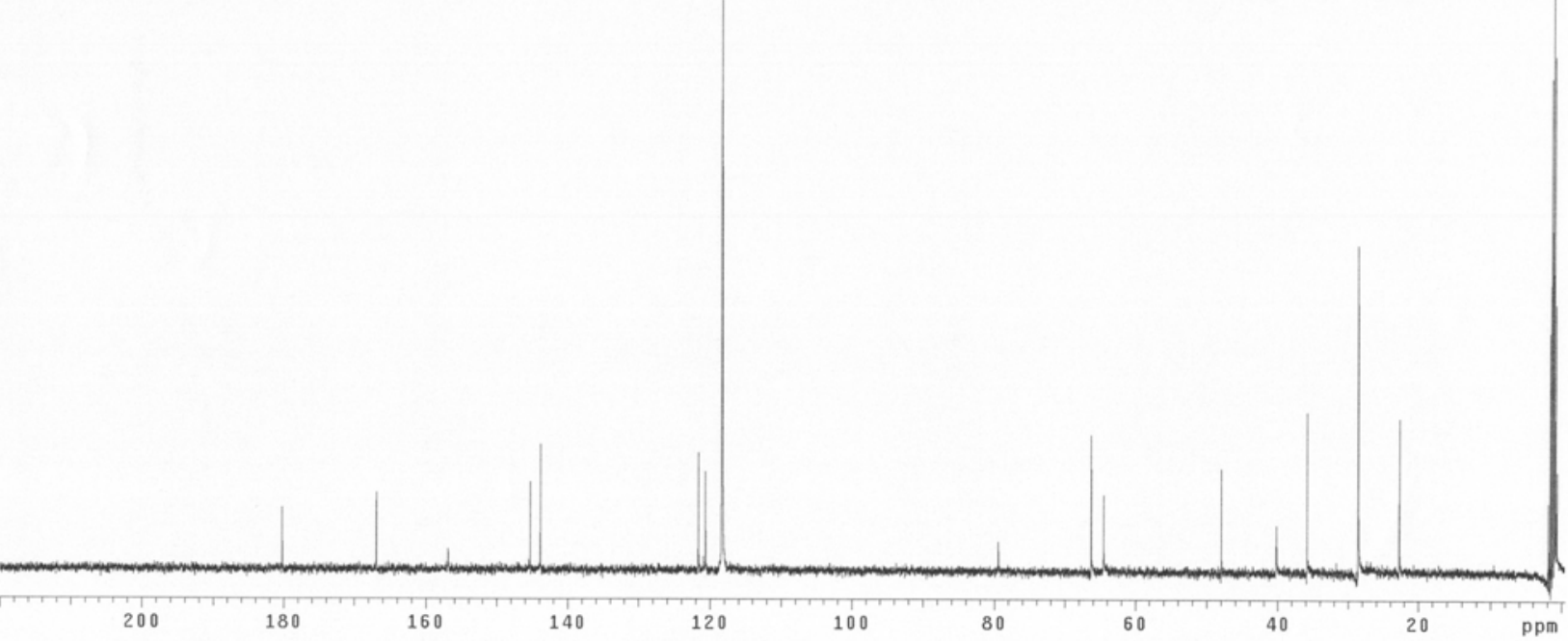


Table 4 Entry 7

JP596 CD3CN-Benzene

Archive directory: /export/home/auto/vnmrsys/data
Sample directory:

Pulse Sequence: s2pul

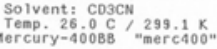

Relax. delay 1.000 sec

Pulse 45.0 degrees

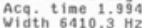

OBSERVE H1, 400.4719454 MHZ

DATA PROCESSING
TT 512065536
Total time 0 min, $50 \mathrm{sec}$

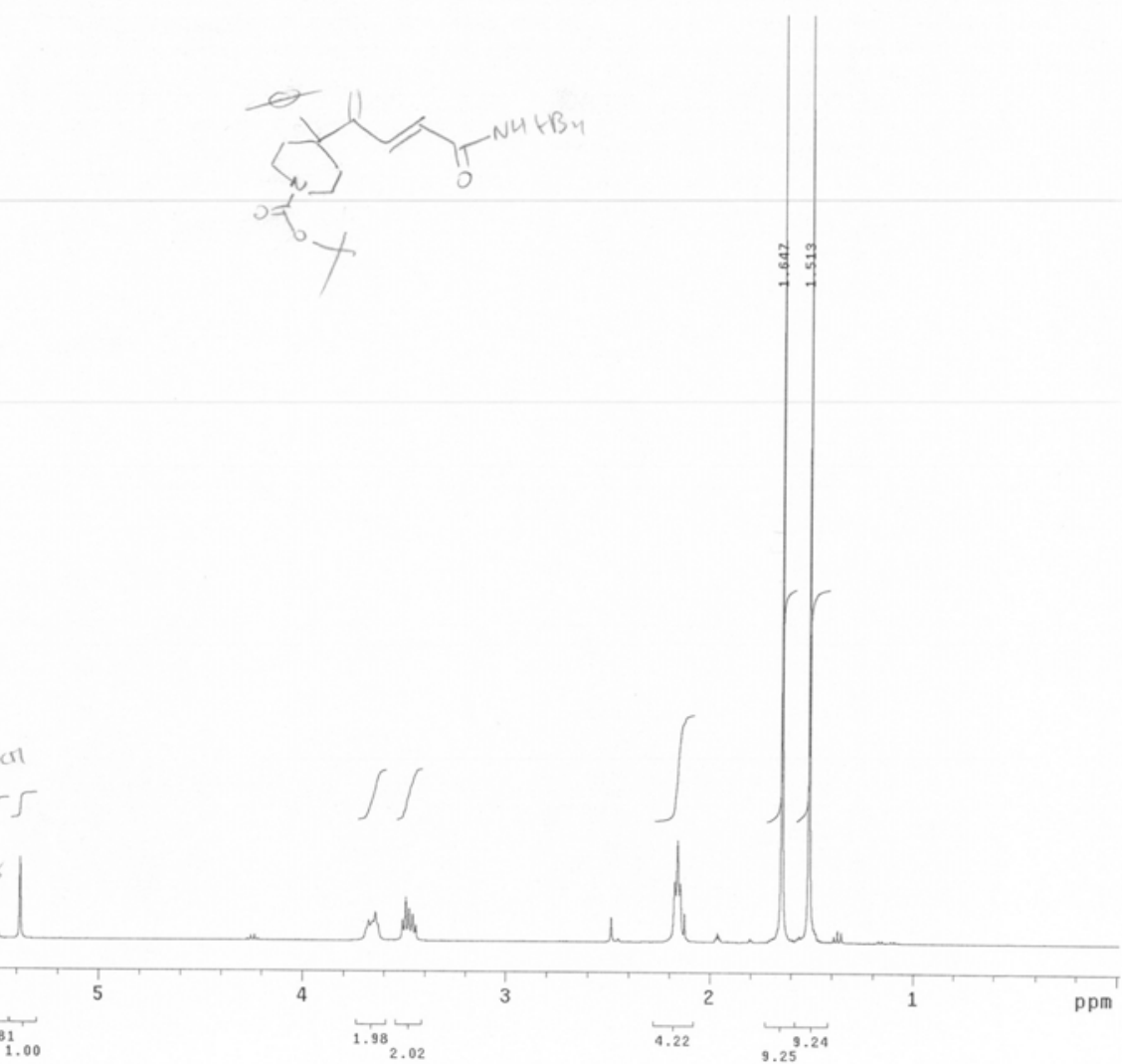

P596 CO3CN-Benzene

Archive directory: /export/home/auto/vnarsys/data
Sample directory:

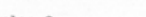

Solvent: $\operatorname{Cos} \mathrm{CN}$ s2

horcury-4008B "merc40

Relax. delay 1.000

Acg time $1.199 \mathrm{sec}$

OBSEVE C13, 100.6987350 MHZ
OECOUPLE H1 400.4738934 MHZ

cont inuous ly on
vaAT $T$-16

Line broadening 1.0 Hz

T) Size 65536 .

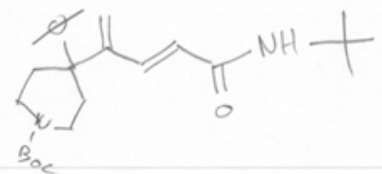

$\min , 46 \mathrm{sec}$

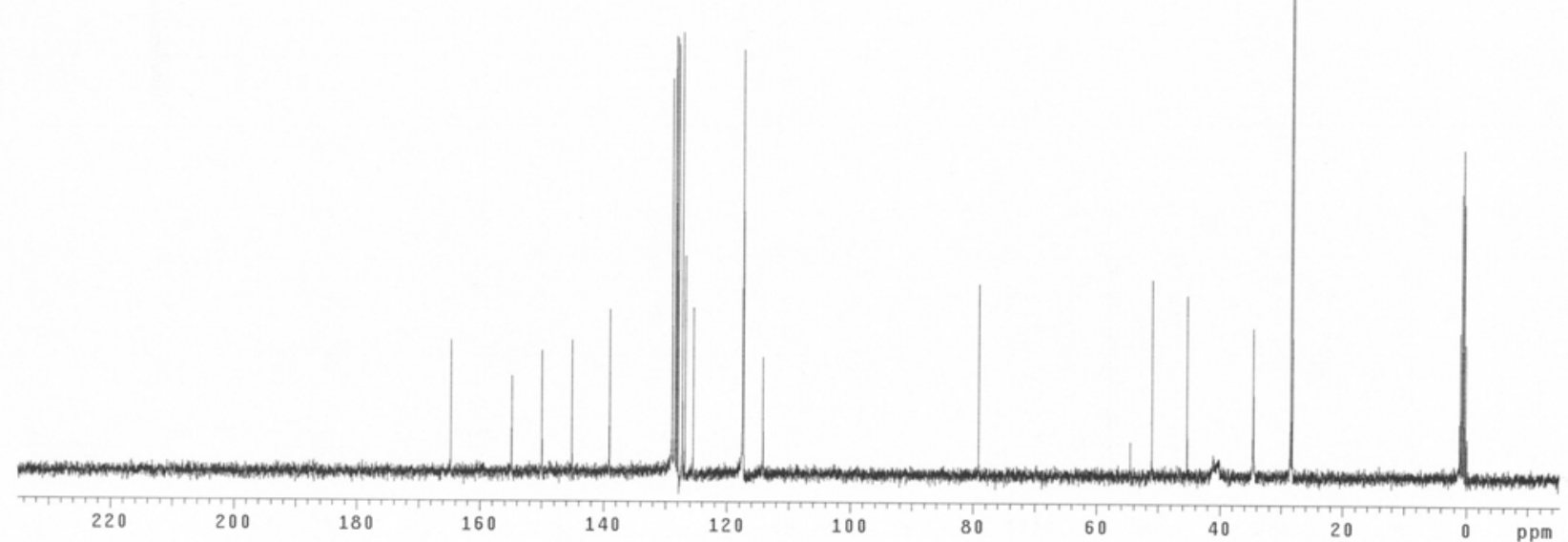


Table 4 Entry 8

793

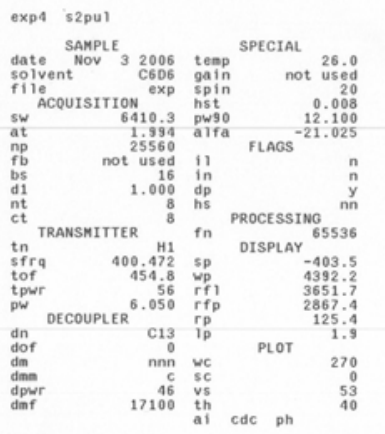

TBDMSO $x^{11}{ }^{N}+$

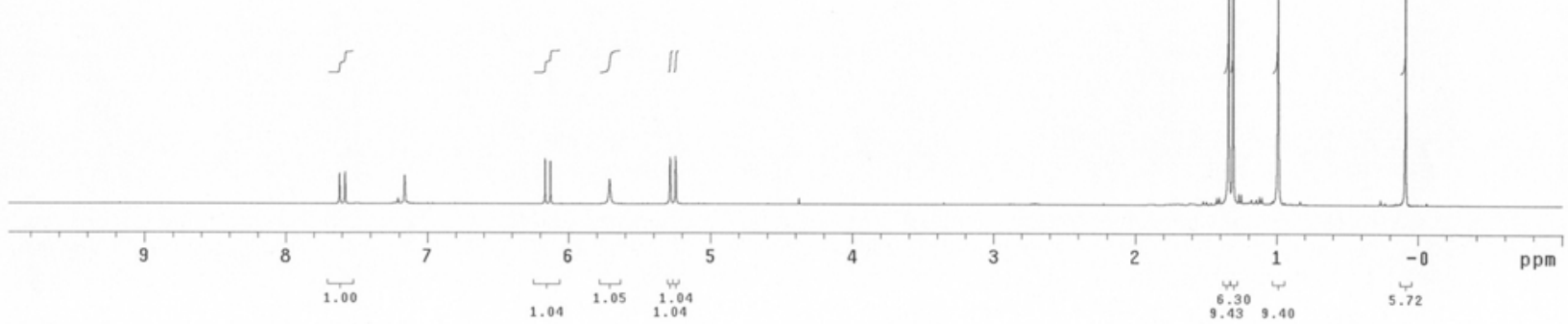

793

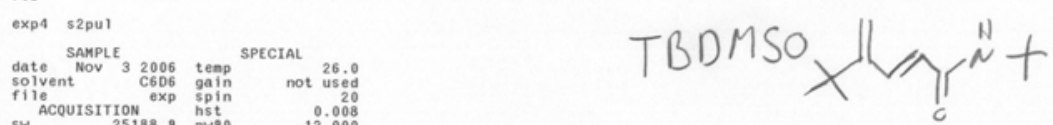

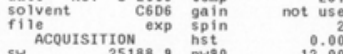

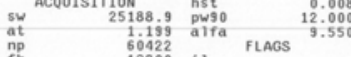

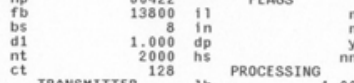

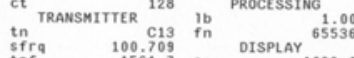

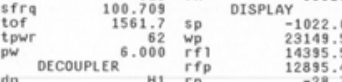

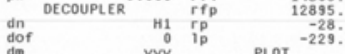

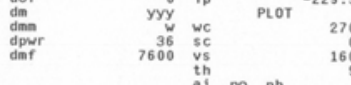

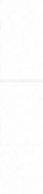


Table 4 Entry 9

JP695-1

Archive directory: /export/home/auto/vnarsys/dato
Sample directory

ile: PROTON

Pulse Sequence: s2pul

Solvent: C606
Temp $26.0 \mathrm{C} / 299.1 \mathrm{~K}$
Nercury-400B

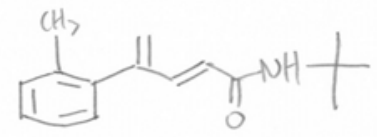

Relax. de lay $1.000 \mathrm{sec}$

Acg time $1.994 \mathrm{sec}$

Vidth 6410.3 H.

OBSERVE H1 400.4698105 MHZ

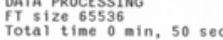
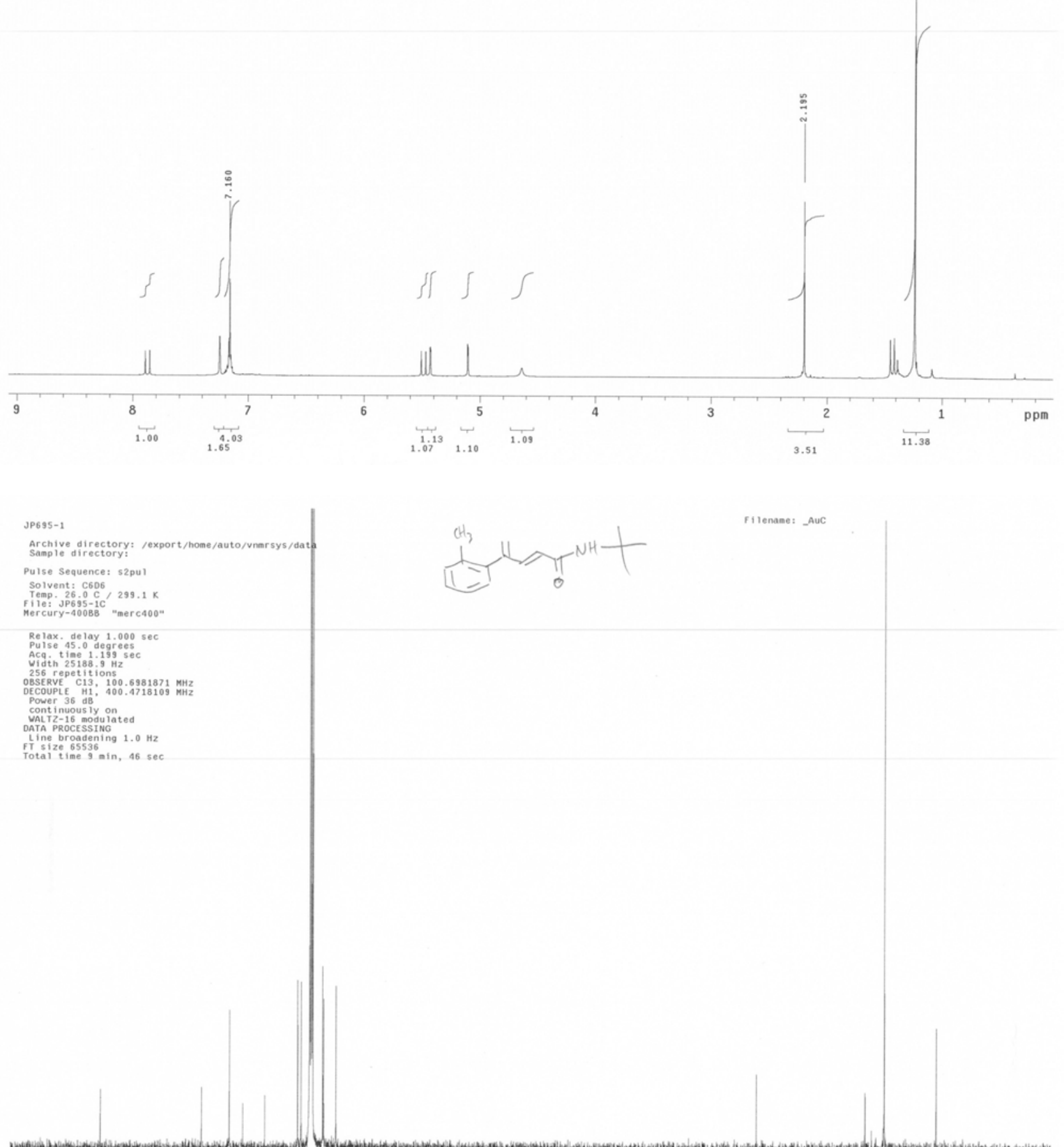

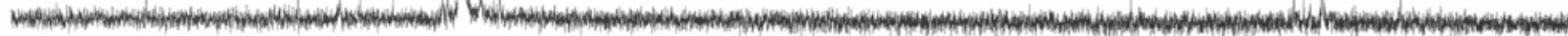


Table 4 Entry 10

JP715

Archive directory: /export/home/auto/vnmersys/data

Sample directory:
File: PROTON

Pulse Sequence: s2pui

III

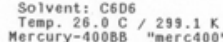

Relax. de lay $1.000 \mathrm{sec}$

Acg. time $1.994 \mathrm{gec}$

16 repetitions

400,4698438

FT size 65536 S

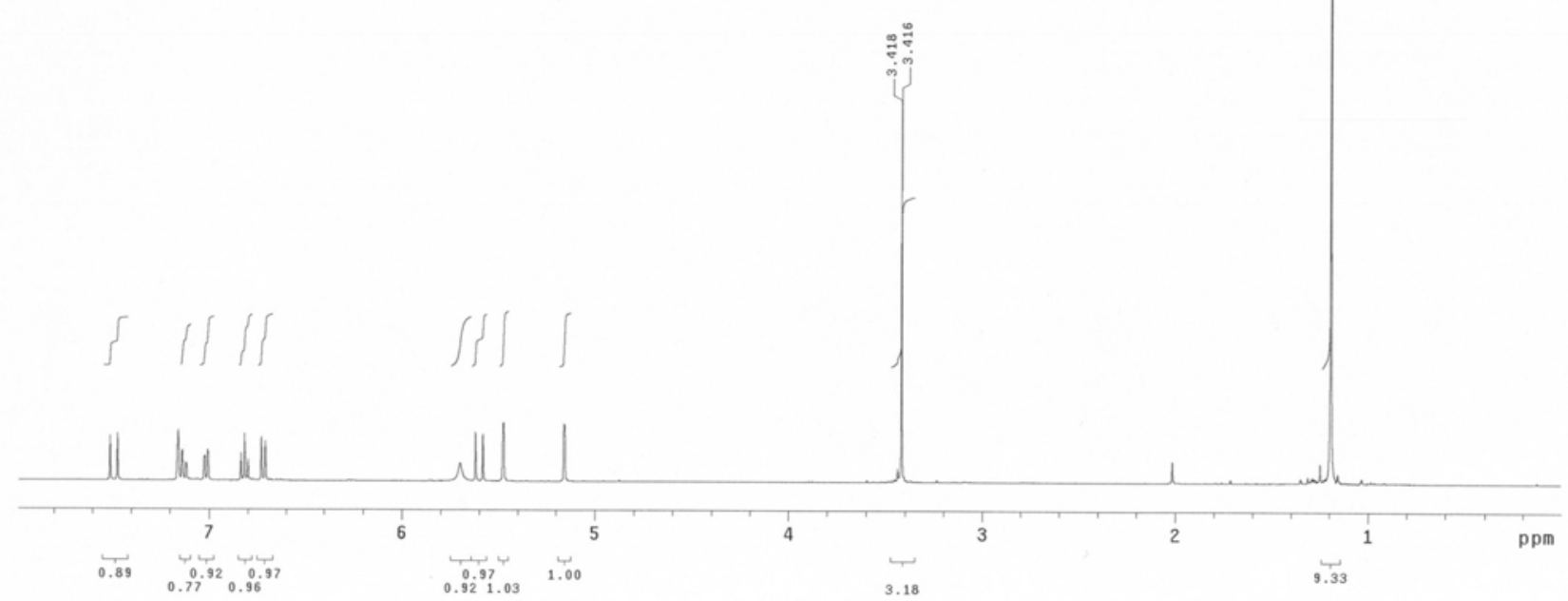

JP715

Archive directory: /export/home/auto/vnmrsys/data
Sample directory:

Sample directory
FIle: CARBON

Pulse Sequence: s2pul

Solvent: C606 $299.1 \mathrm{~K}$

Relax. de lay 1.000 sec

Pulse 45.0 degrees

Widin $25188: 9 \mathrm{~Hz}$

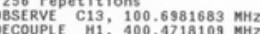

Continuous ly on
WALTZ-16 modulated

ine broadening $1.0 \mathrm{~Hz}$

T size 65536 .

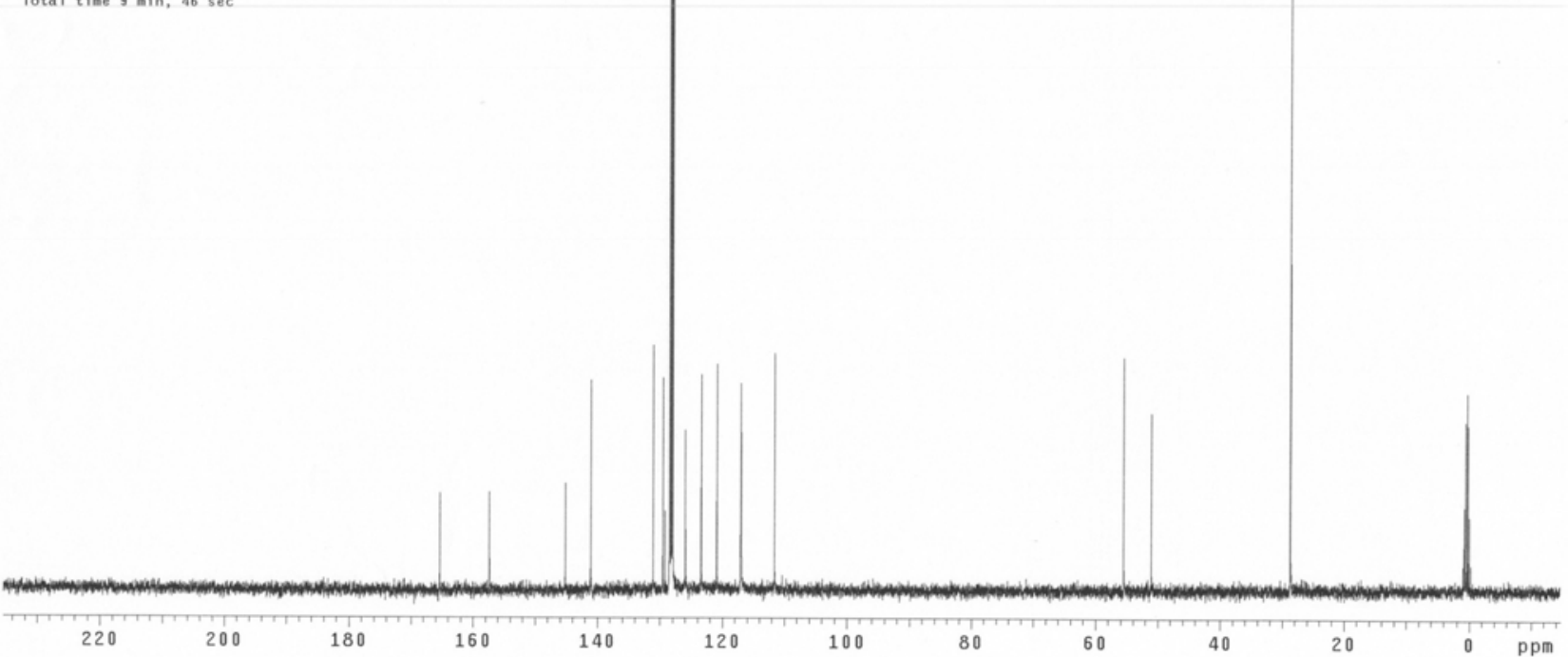


Table 4 Entry 11

JP434

Archive directory: /export/home/auto/vnarsys/data
Sample directory:

Pulse Sequence: 52 pul
Solvent: C6D6
Temp, 26.069 .10

Solvent: C606
Temp. $26.0 \mathrm{C} / 299.1 \mathrm{~K}$
Mercury -4008 "merc400"

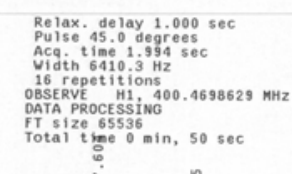

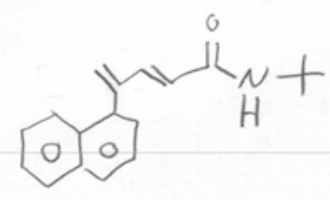

$\mid$

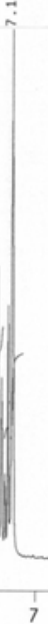

$\begin{array}{cc}0.86 .36 & 1.91 .44 \\ 1.27 & 0.69 .66\end{array}$

$1.00^{0.90 .96} \underbrace{0.99}_{0.99}$

JP 434

JP434
Archive directory: /export/home/auto/vnmersys/dafa
Sample directory:
File: CARBON

Pulse Sequence: spur

Solvent: C606
Temp. $26.0 \mathrm{C}$, $299.1 \mathrm{~K}$
Mercury -40088 "merc400"

Relax. delay $1.000 \mathrm{sec}$
Pulse 45.0 degrees

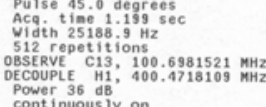

Cont inuousiy on
WAT Z-16 modulated
DATA PROCESSING
Line broadening $1.0 \mathrm{~Hz}$
FT 5 size 65536 size
Total time $19 \mathrm{~min}, 32 \mathrm{sec}$

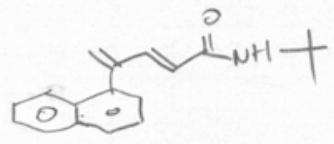

60

S 21 
Table 5 Entry 1

JP678-pure

Archive directory: /export/home/auto/vnmrsys/data
Sample directory:
File: PROTON

Pulse Sequence: s2pul

Solvent: CD3CN
Temp. $26.0 \mathrm{C}$. $299.1 \mathrm{~K}$
Mercury-400BB "merc400"

Relax. delay $1.000 \mathrm{sec}$
Pulse 45 . 0 degrees

Acq. time $1.994 \mathrm{sec}$

16 repetitions
OBSERVE

DATA PROCESSING

TT size 65536
Total time 0 min, $50 \mathrm{sec}$
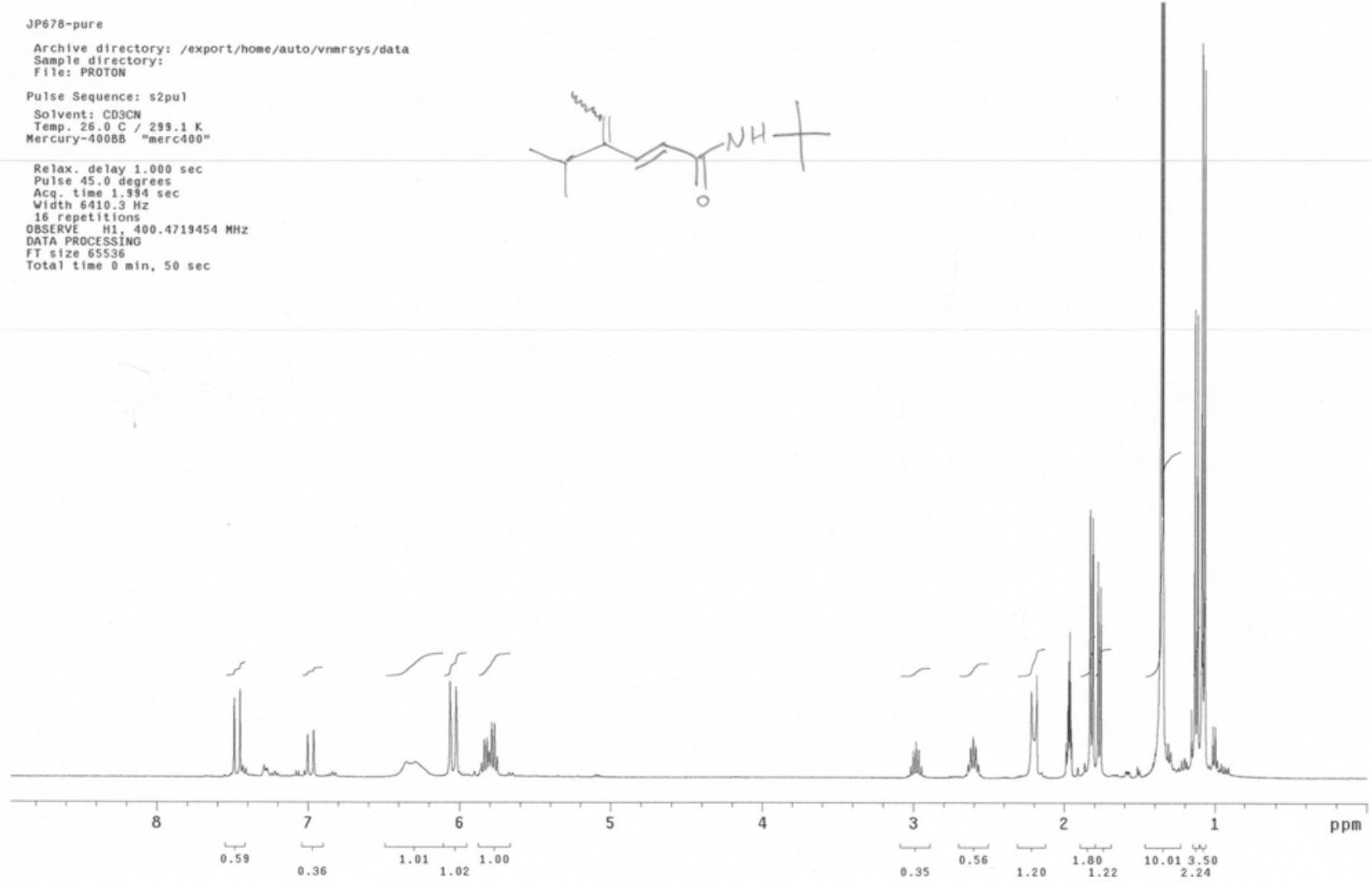

Archive directory: /export/home/auto/vnarsys/data
Sample directory:

Pulse Sequence: s2pul

Solvent: CDC13 $299.1 \mathrm{~K}$

File: $J P 678 \mathrm{C}$
Mercury-400BB "merc 400

Re lax. delay $1.000 \mathrm{sec}$
Pulse 45 . O degrees

Acq time $1.199 \mathrm{sec}$

18112 repetitions
OBSERVE C13, 100.6986529 MHZ

DECOUPLE H1, 400.4717749

Cont inuous ly on

ATA PROCESSING

FT size $655365 \mathrm{~s}$
Total tine $63 \mathrm{hr}, 36 \mathrm{gin}, 17 \mathrm{sec}$

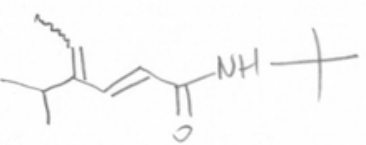


Table 5 Entry 2

JP889-1

Archive directory: /export/home/auto/vnnrsys/data

Sample directory
File: PROTON

ence: 52 pu

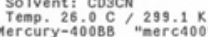

Relax. de lay $1.000 \mathrm{sec}$
Pulse. 45.0 degrees
Acq. tine 1.9944 sec

Width $6410.3 \mathrm{H}$

OBSERVE H1 400.4719454 MHZ

PT size 65536
Total time 0 min, $50 \mathrm{sec}$
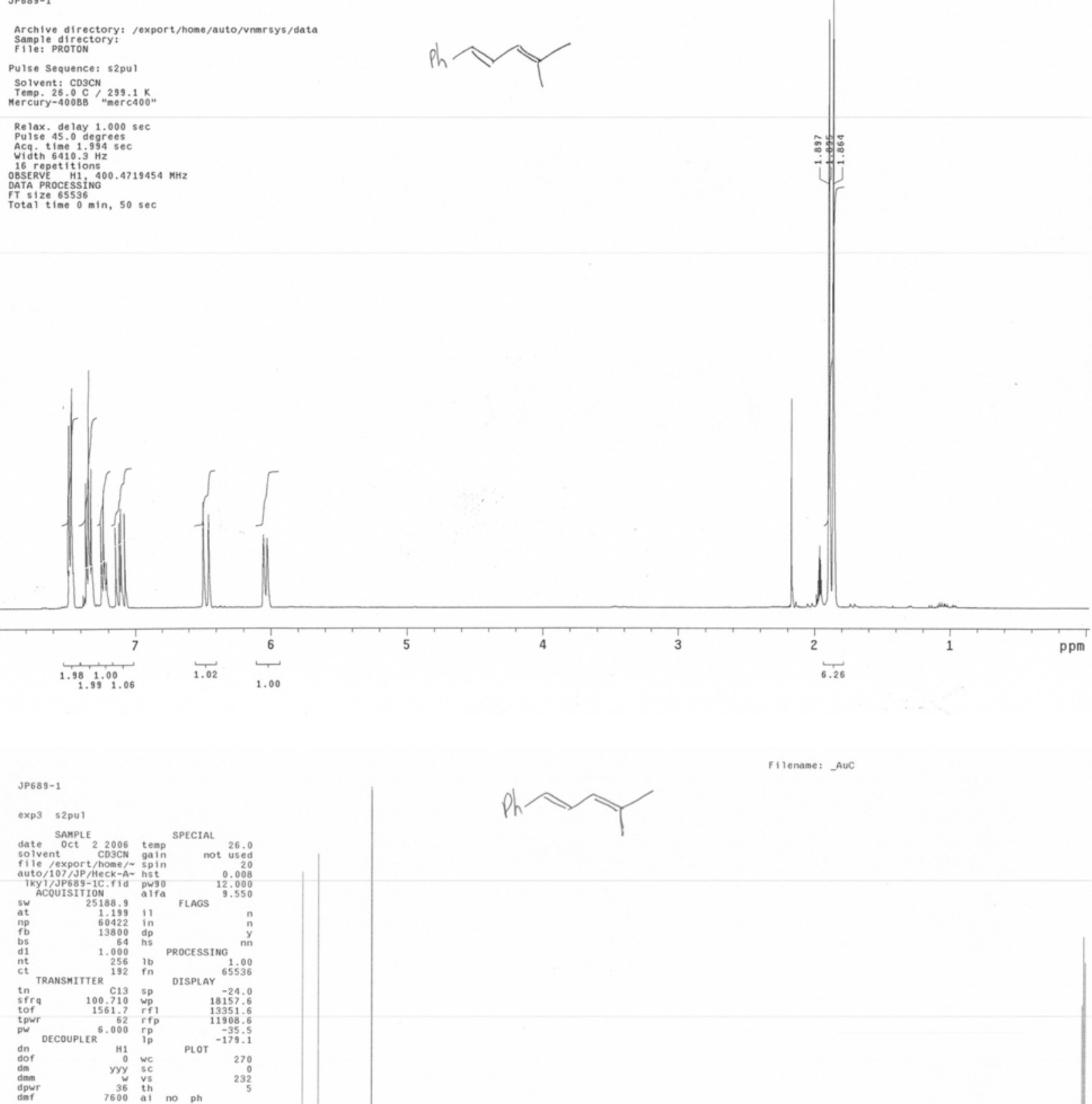

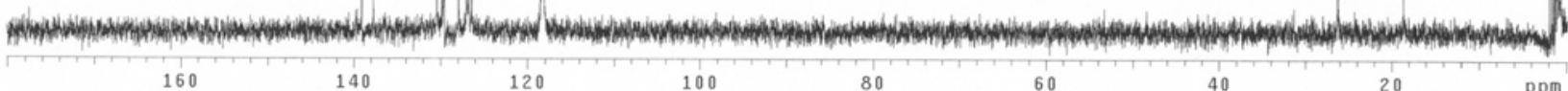


Table 5 Entry 2

Archive directory: /export/home/auto/vnarsys/data
Sample directory:

Pulse Sequence: s2pu

Solvent: $\mathrm{CD}^{\mathrm{C} C N}, 299.1 \mathrm{~K}$

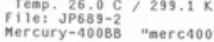

Relax. delay $1.000 \mathrm{sec}$
Puise 45.0 degrees

Aca time 1.994 sec

16 repetitions
OBSERE

OESERVE H11 400.4719454
OATA PRoCESSING
FT SIZE 65536

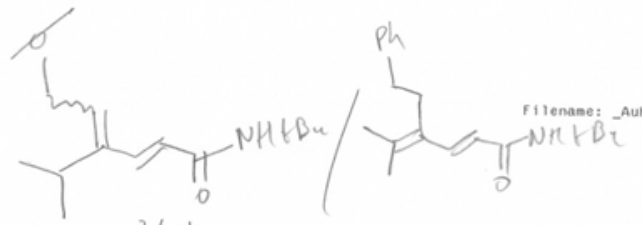

Total time 0 min, 50 sec

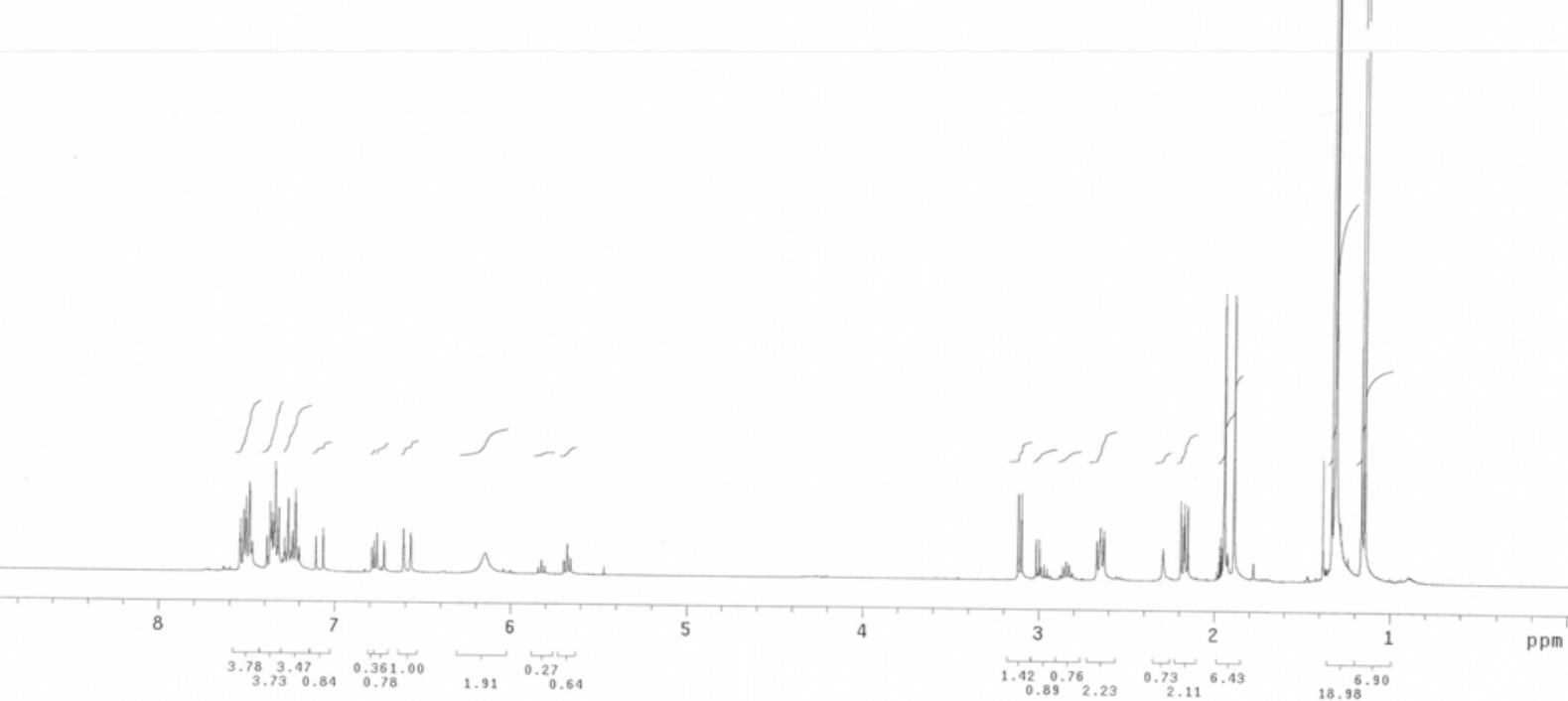

JP689-2pure

Archive directory: /export/home/auto/vmmrsys/data

sample directory:

Pulse Sequence: 2 pul

Solvent: $\mathrm{CD} 3 \mathrm{CN}$

Tamp. $26.0 \mathrm{C} / 299.1 \mathrm{~K}$

Mereury-400BB ${ }^{-m e r c 400 *}$

Relax. Aelay $1.000 \mathrm{sec}$

Pulse 45.0 degrees

wed. the 1.199 sec

22080 repetitio

OBSERVE C13, $100.6986529 \mathrm{~kg}$

DECOUPLE H1, 400.4738934 NH:

Power $36 \mathrm{~dB}$

continuousiy on

WALTZ-16 modulated

DATX Processing

(1)

Total time $63 \mathrm{hr}, 36 \mathrm{~min}, 17 \mathrm{sec}$

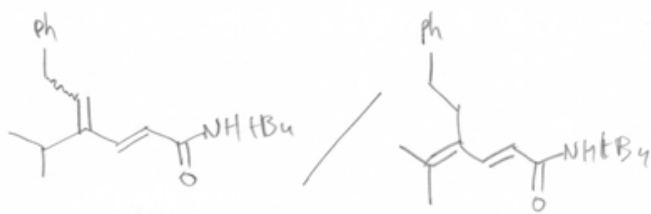


Table 5 Entry 3

$\begin{array}{ll}767 & \\ \text { exp7 } & \text { 52pul }\end{array}$

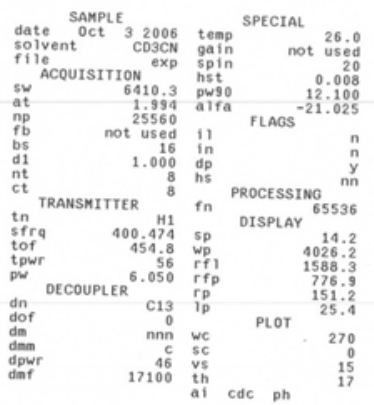
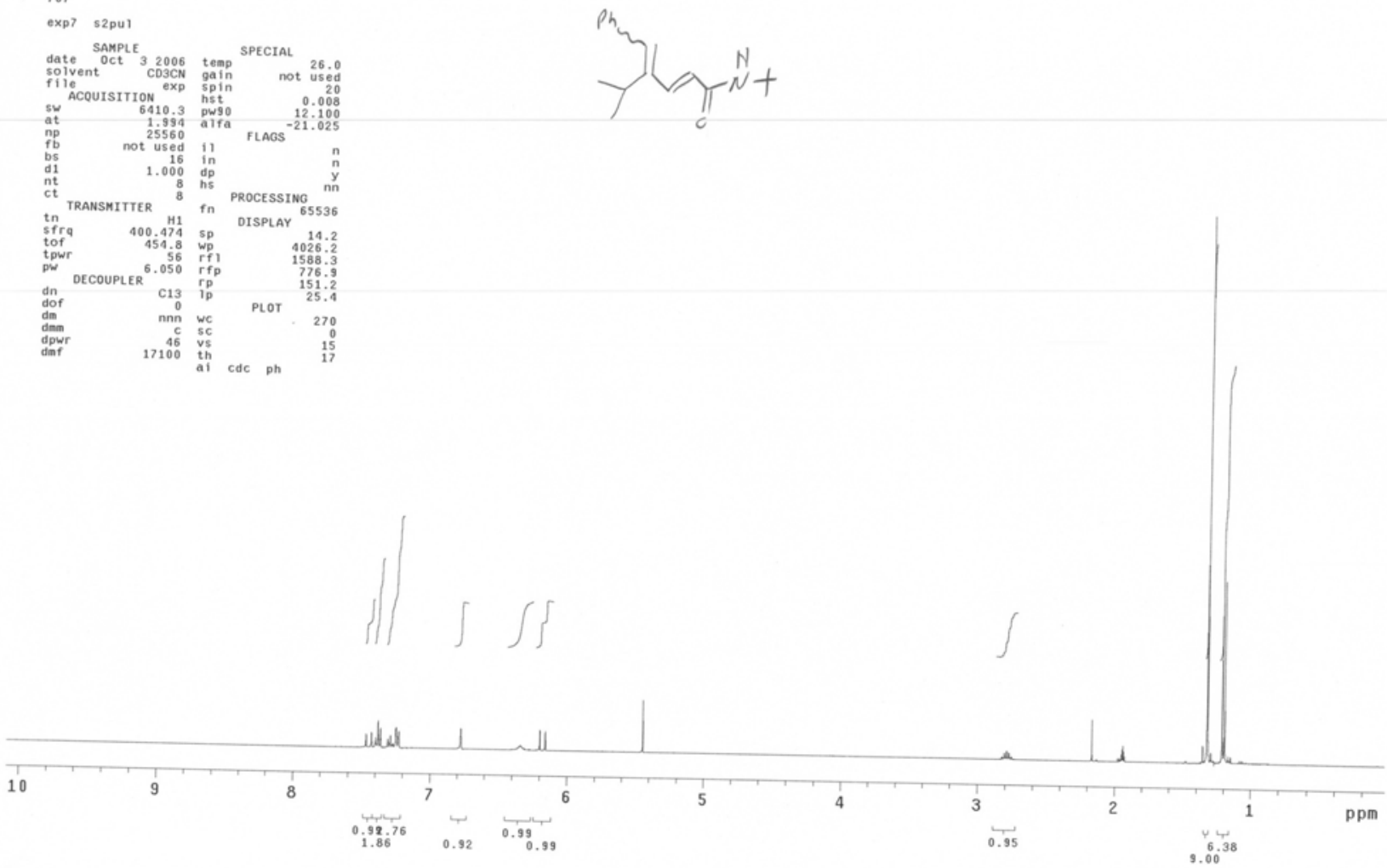

$\begin{array}{ll}767 & \\ \exp 7 & \text { s2pul }\end{array}$
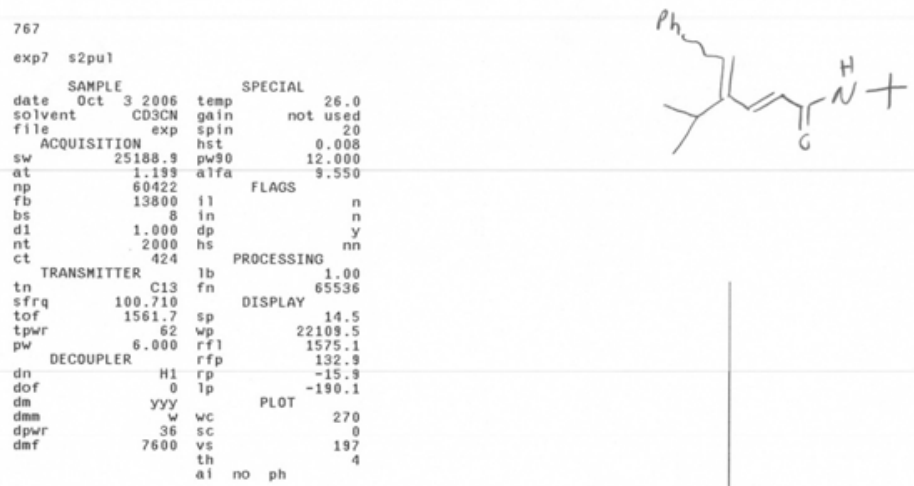

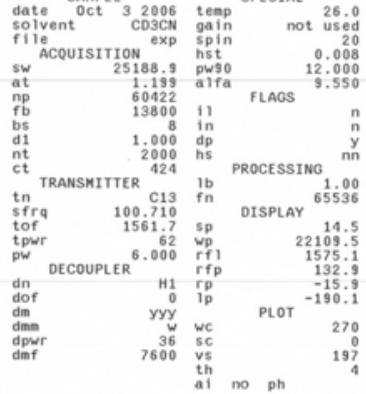

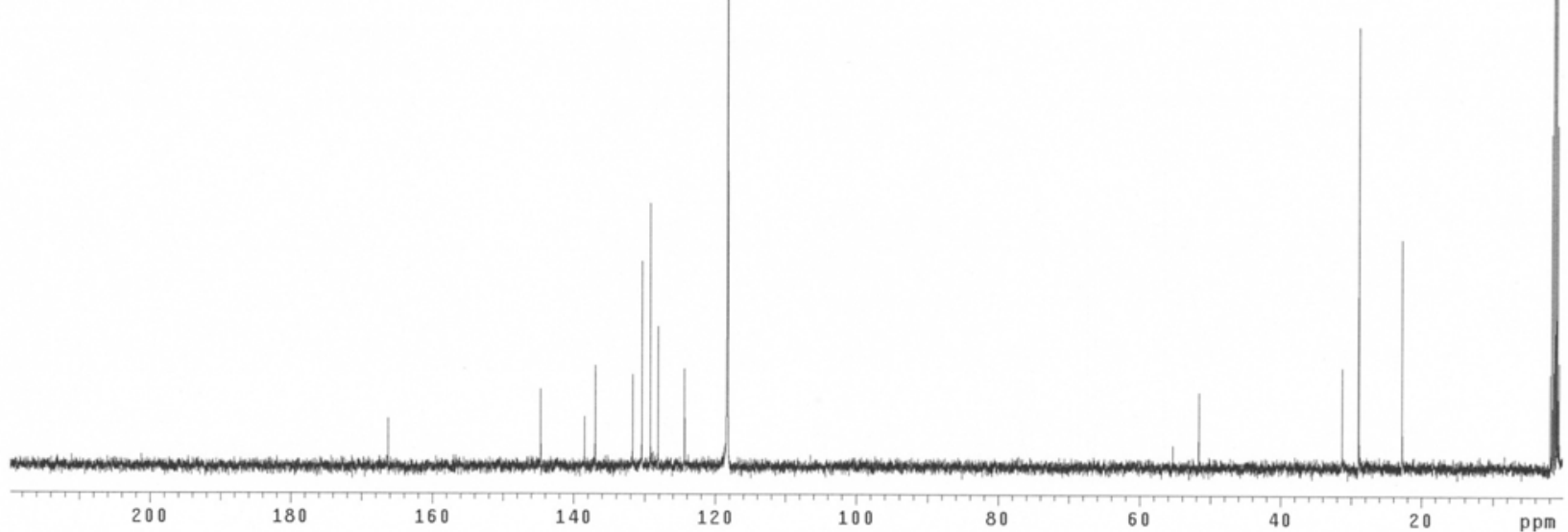


Table 5 Entry 4

JP716

Archive directory: /export/home/auto/vnmrsys/date IIle: PROTON

Pulse Sequence: s2pul

Solvent: C606
Temp. $26.00 \mathrm{C} / 299.1 \mathrm{~K}$
Mercury-400B

Relax. de lay $1.000 \mathrm{sec}$

Relax. delay 1.000 sec
Pulse 45.0 degrees
Acq. 1 ime $1.994 \mathrm{sec}$

Acatin $6410.3 \mathrm{~Hz}$

16
BSERVE H1,

DATA PROCESSING
FT S SIZ 65536
Tot

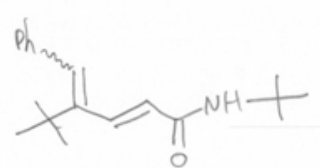

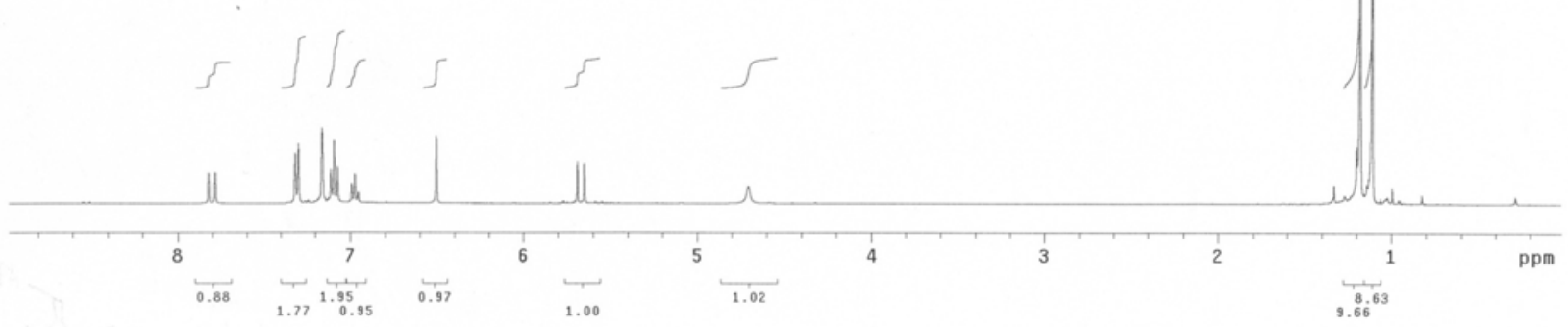

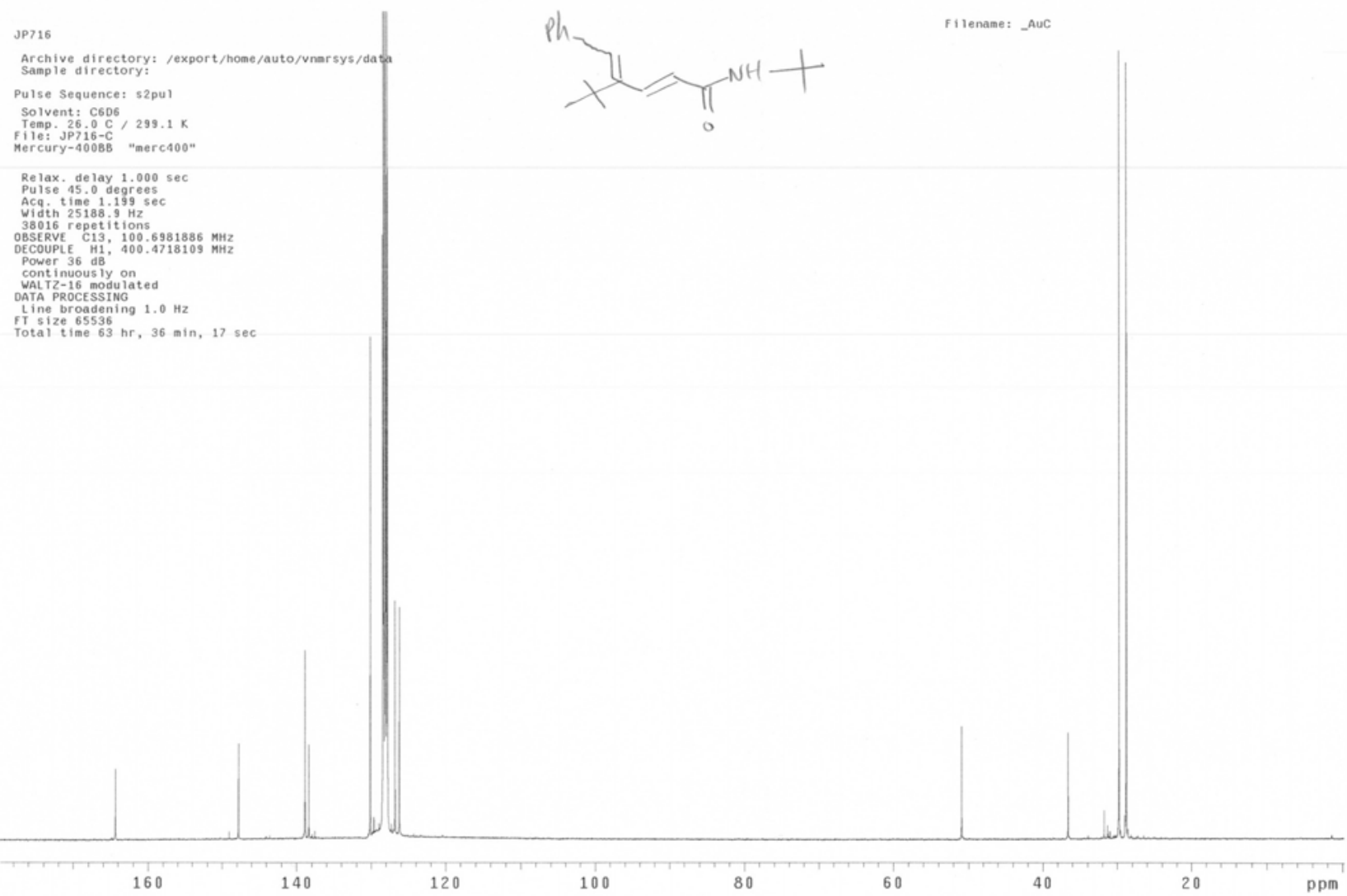


Table 5 Entry 5 (Z Isomer)
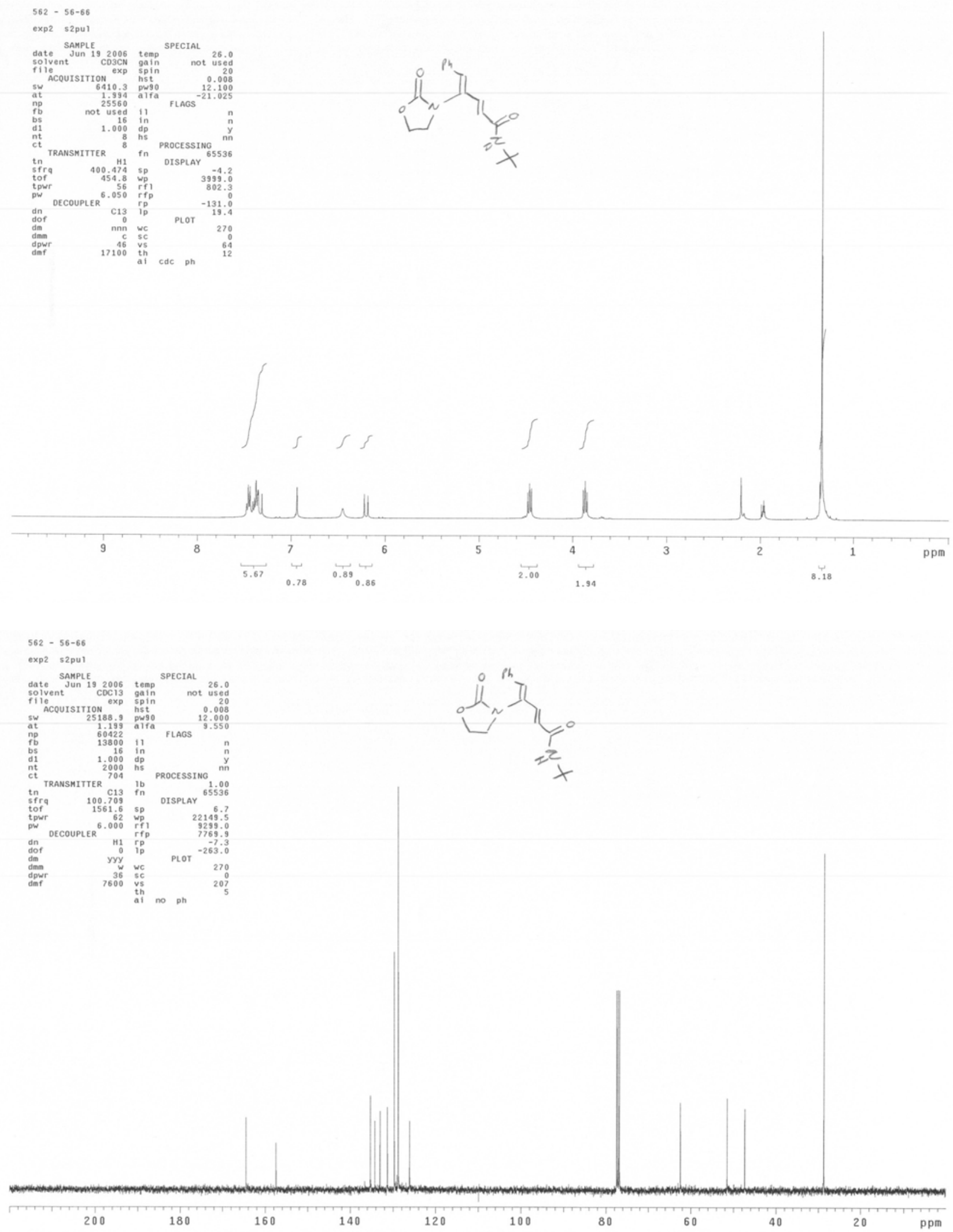
Table 5 Entry 5 (E Isomer)

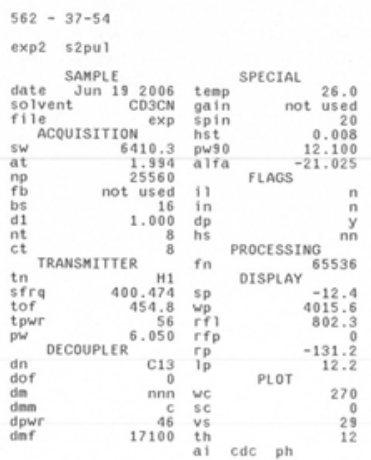
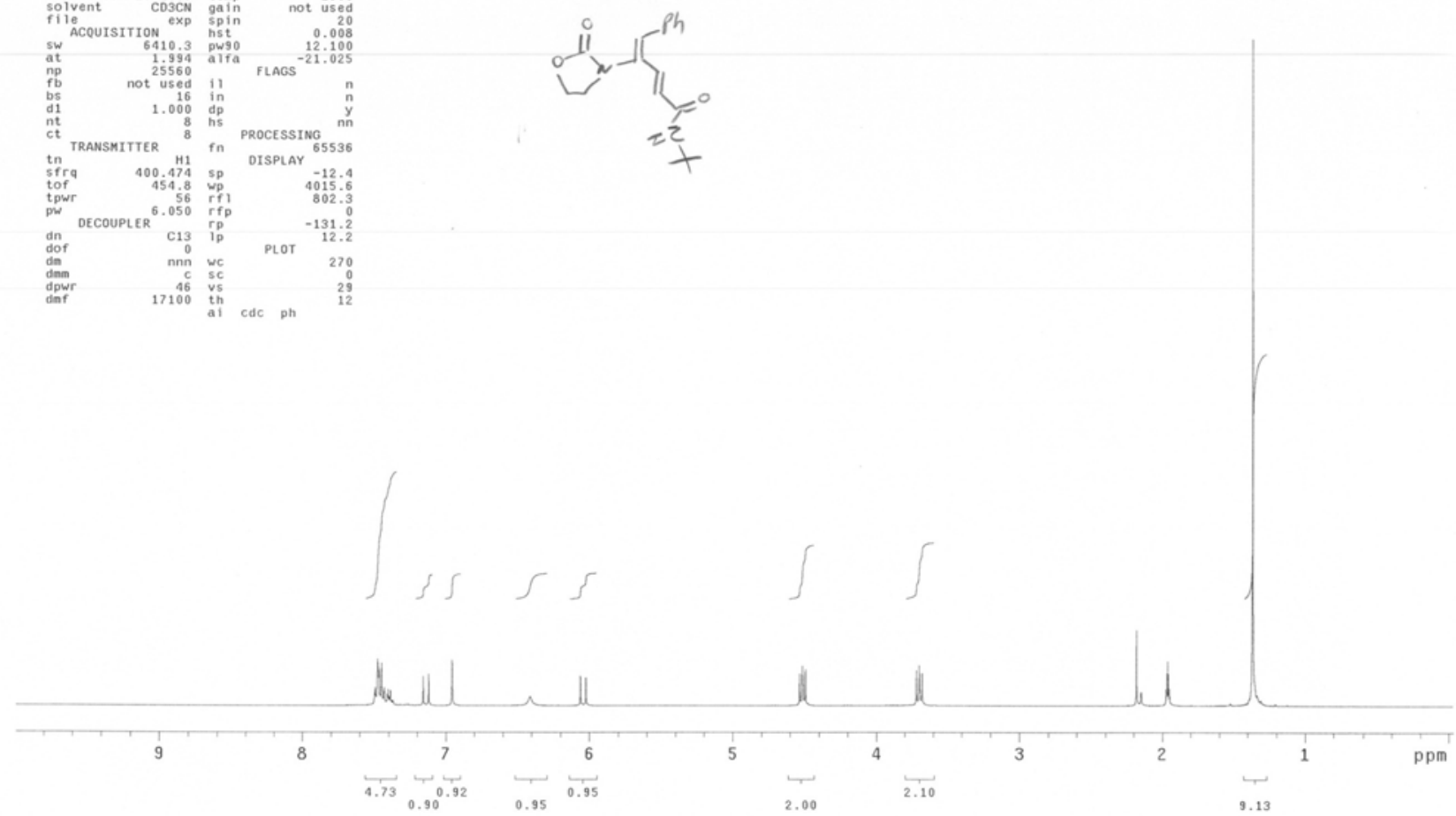

652-37-52

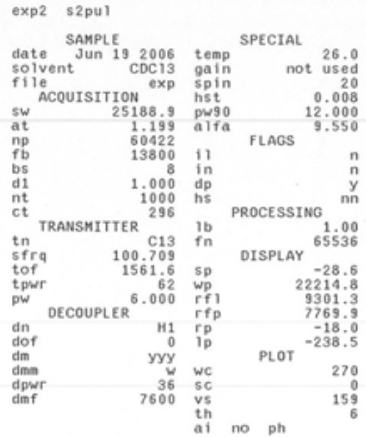
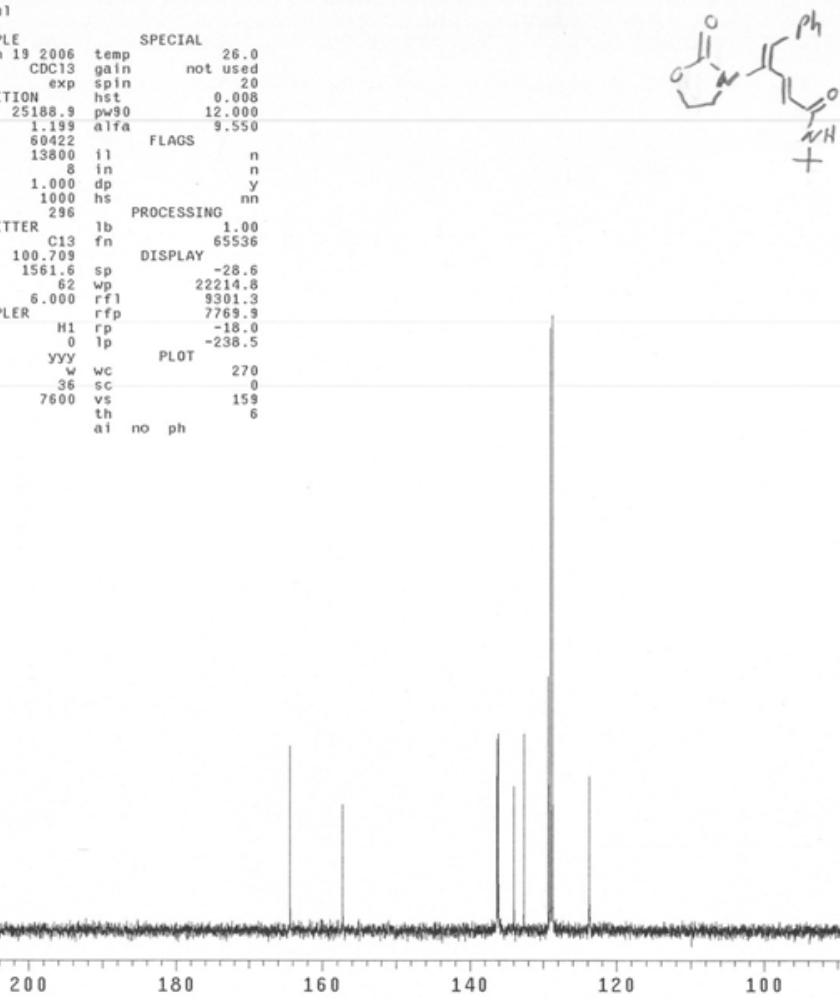

60

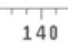

40

120

100

80

60

40

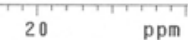


Table 6 Entry 1

JP590

Archive directory: /export/home/auto/vnarsys/data

Archive directory:
Sample directory:
Fille: PRoTon

Pulse Sequence: s2pul

Solvent: C606
Temp. $26.6 \mathrm{C} / 299.1 \mathrm{~K}$
Mercury-4008B "

Relax. de lay $1.000 \mathrm{sec}$

Acq. tine 1.994 se

16 repetitions

$0.4698442 \mathrm{MHZ}$

DATA PROCESSING
FT 512 ize 65536 .
Total time 0 min, 50 sec

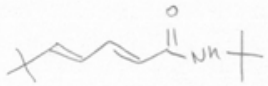

JP590

Archive directory: /export/home/auto/vnarsys/data

Saaple directory
File: CARBON

Pulse sequence: s2pur

Solvent: C606
Temp. $26.06 \mathrm{C}, 299.1 \mathrm{~K}$
Mercury $=400 \mathrm{CB}$ " "merc400"

Relax. delay $1.000 \mathrm{sec}$
Pulse 45.0 degrees

Act time 1.1995

256 repetitions
OBSERVE C13, 100.6981506
MHZ

OESERVE C13, 100.6981506
DECOUPLE H1 400.4718109

continuous ly on
waLt $2-16$ modulated
chin

DAA PRocESSing $1.0 \mathrm{~Hz}$

FT size 65536 .

id vill

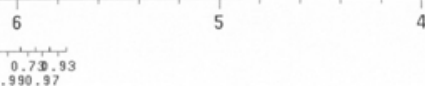

3

2

$\underbrace{1}_{8.22}$

990.9
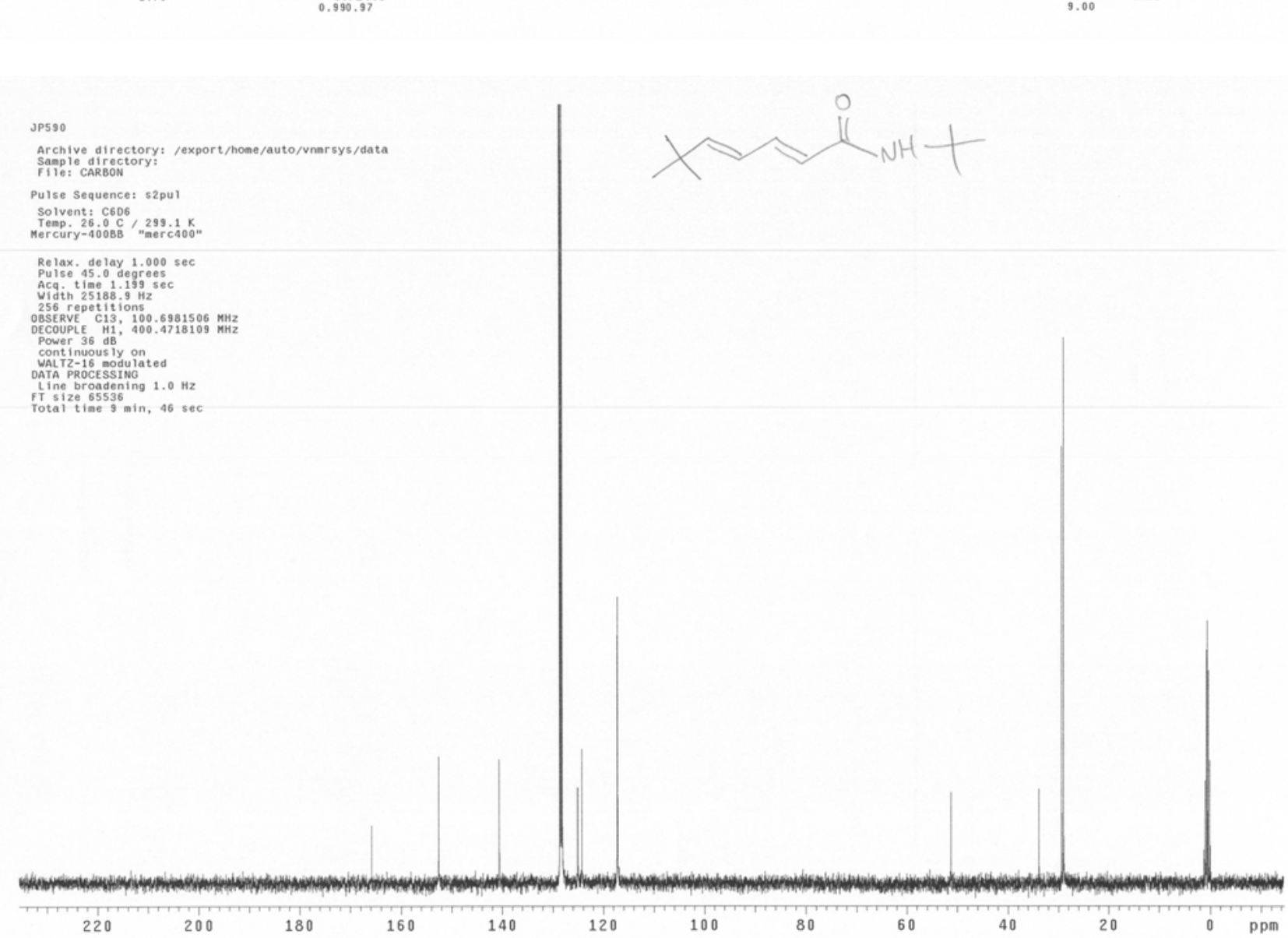
Table 6 Entry 2

JP586-pure-C03CN+Benzen

Archive directory: /export/home/auto/vnarsys/data

Sample director
File: proton

Pulse Sequence: s2pul

Solvent: C606
Temp. $26.0 \mathrm{C} / 299.1 \mathrm{~K}$
Mercury-4008B

Relax. delay 1,000 sec
Pulse 45 . degrees

Acs $\operatorname{time} 1.994$

16 repet itions
OBSERVE
HI $400.4698436 \mathrm{MHZ}$

OBSERVE H1
OA
OATA PROCE

Total time $0 \mathrm{~min}, 50 \mathrm{sec}$

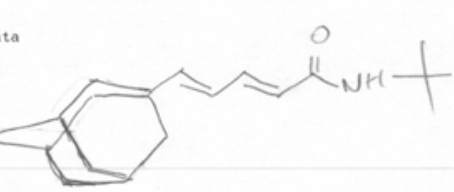

Archive directory: /export/home/auto/vnarsys/data

Sample directory:
file: CARBON

Pulse Sequence: s2pul

Solvent: C606
Temp. $26.0 \mathrm{C}$ C $299.1 \mathrm{~K}$.
Mercury $400 \mathrm{~B}$ "

Relax. delay 1,000 sec

Acq tine 1.199 sec

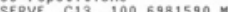

COUPLE H1, 400.4718109 MH2

cont inuous Iy on
walt $T 2-16$ nodulated

Line broadening $1.0 \mathrm{~Hz}$

tize 65536 (2)
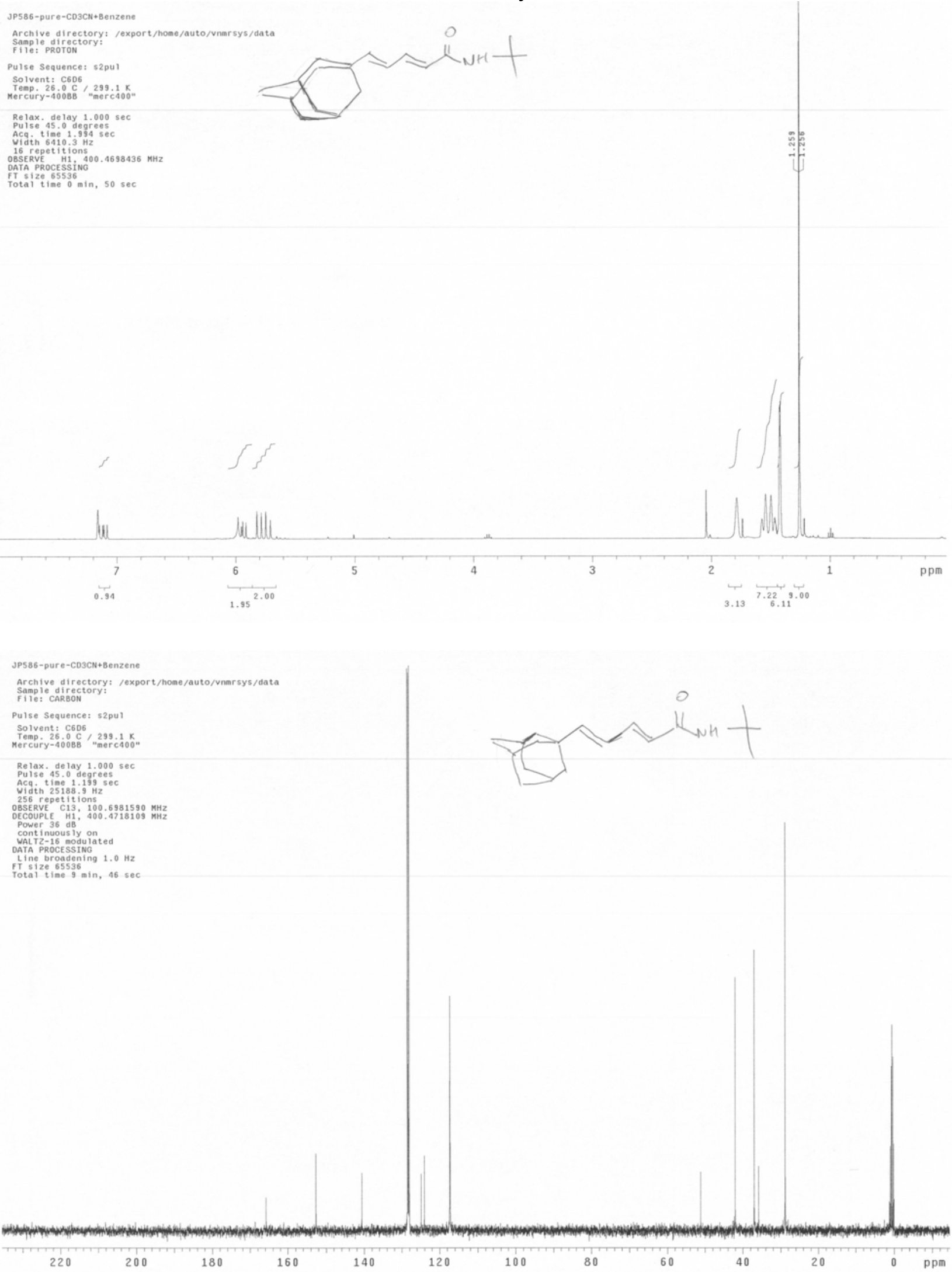
Table 6 Entry 3

JP5 97

Archive directory: /export/home/auto/vnmisys/data

File: PROTON

1se Sequence: s2pul

Solvent: C606
Temp. $26.0{ }^{\circ} \mathrm{C}^{2}, 299.1 \mathrm{~K}$
Mercury-400BB "merc400"

Relax. de lay 1.000 sec

Acq. time 1.994 sec

Width 6410.3 H

OBSERVE H1, 400.4698436 MHI

FT size 65536
Total time 0 in, $50 \mathrm{sec}$

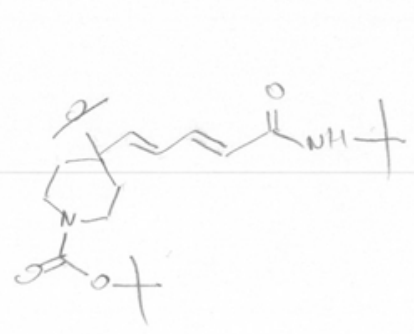

Archive directory: /export/home/auto/vnersys/data

Sample directory
file: CARBON

Pulse Sequence: s2pul

Solvent: C6D6
Temp. $26.0 \mathrm{C}$ C $299.1 \mathrm{~K}$
Mercury-400BB" "merc400"

Relax. de lay $1.000 \mathrm{sec}$

Acg. time 1.199 sec

OBSERVE C13, 100.6982031
MHZ

Power $36 \mathrm{~dB}$
continuous iy on

ATA PROCESSING

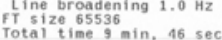

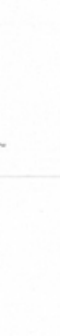


Table 6 Entry 4

JP5 98

Archive directory: /export/home/auto/vnmrsys/dat
Sample directory:

Sample directory
File: PROTON

Pulse Sequence: 52 pul

Solvent: ${ }^{C 03 C N}, 299.1$

Relax. de lay 1.000 sec

Pulse 45.0 degrees

Acg. time 1.9944 sec

OBSERVE H1, 400.4719454 MHz

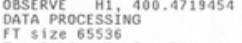

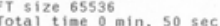

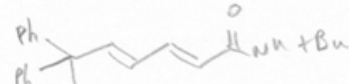

$P$ Lnt $n u$

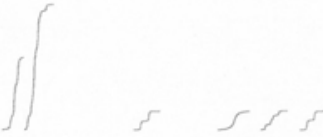

jp598

Archive directory: /export/home/auto/vnarsys/data
Sanple directoryi

Sample directory
File: CARBON

$\phi^{\infty} \sim \mathrm{H}_{\mathrm{Ne}}+1$

Pulse Sequence: 52 pu

Solvent: $C 03 C \mathrm{CN}$
Temp. $26.0 \mathrm{C} / 299.1 \mathrm{~K}$

Re lax. de lay 1.000 sec

Acq. ${ }^{2}$ ime $1.199 \mathrm{sec}$

SERVE C13 100.5987235

PEOUPLE H1, 400.4738934 Mi

cont inuous iy on
vaAT $T$ - 16 modulate

line broadening $1.0 \mathrm{~Hz}$

FT size 655356 . 
Table 6 Entry 5
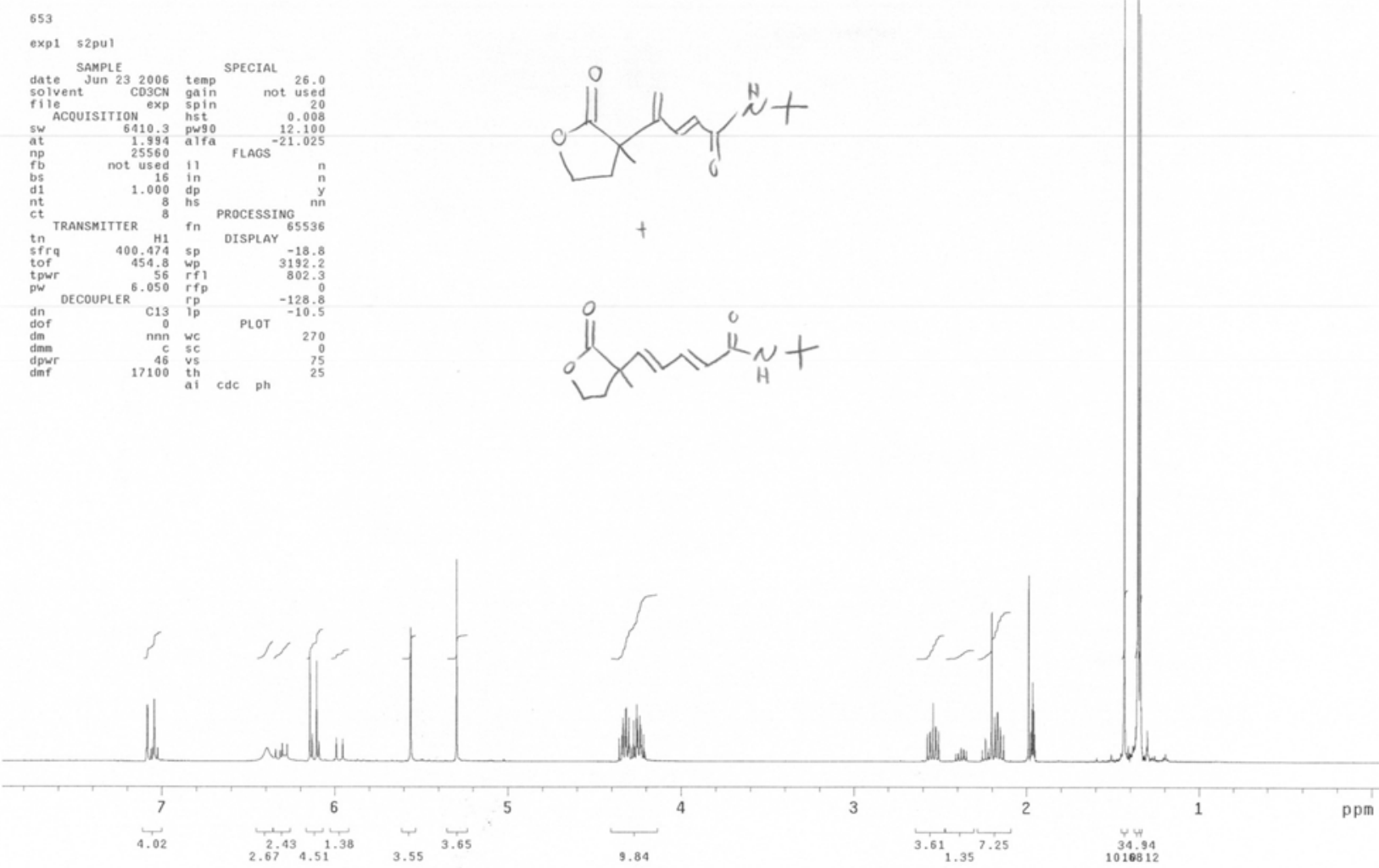
Table 7 Entry 1

JP695-2

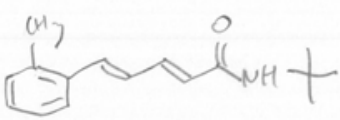

Archive directory:
Sample directory:
File: PRoTON

Pulse Sequence: s2pul

Solvent: C606
Temp. $26.00 \mathrm{C} / 299.1 \mathrm{~K}$
Mercury-400BB

Relax. delay $1.000 \mathrm{sec}$
Puise 45.0 degrees

Acq ${ }^{\text {time }} 1.994$

OBSERVE HI 400.4698442

DATA PROCESSINO

Tize 65536
Total time $00 \mathrm{~min}, 50 \mathrm{sec}$

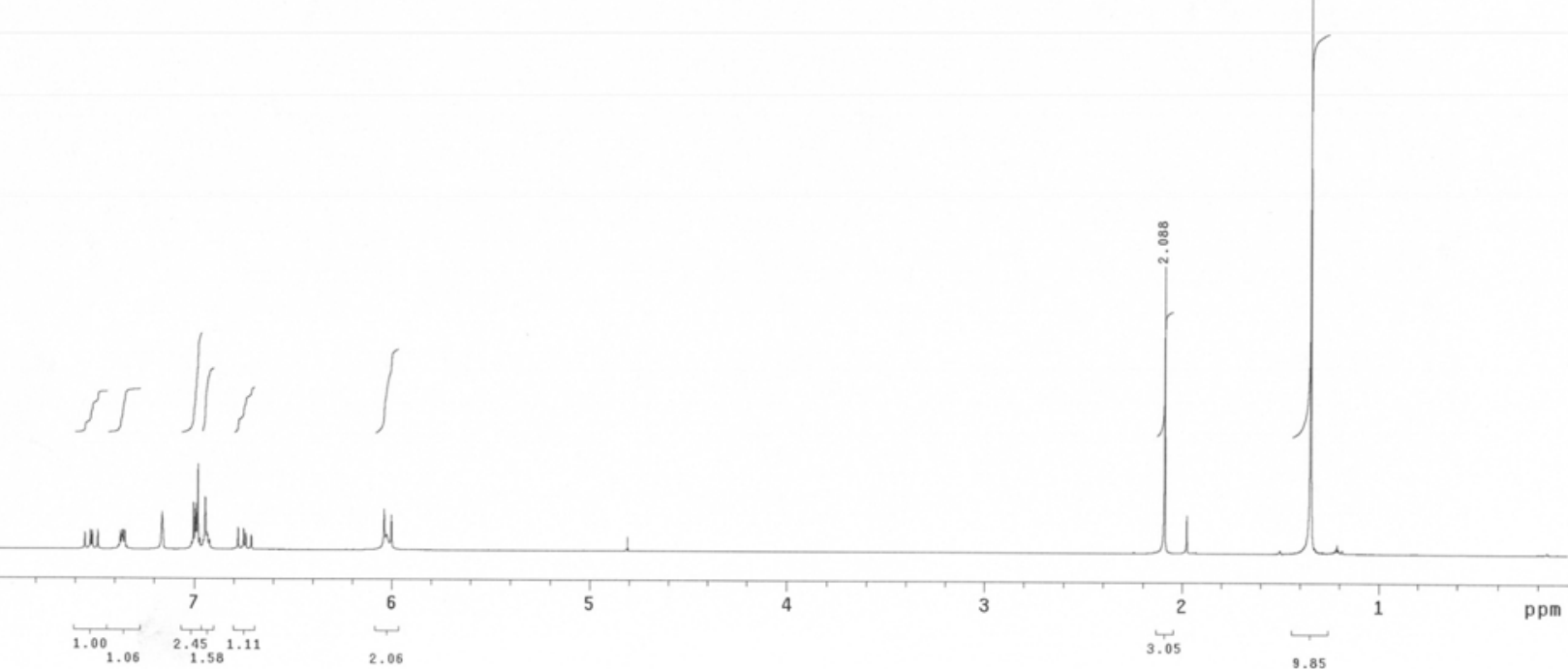

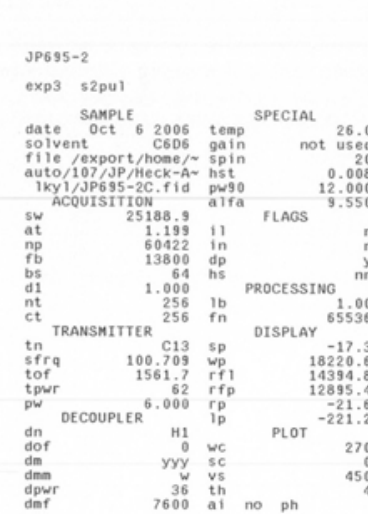

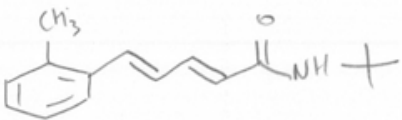

Filename: _AuC

dimf $\quad 7600$ ai no ph 4 
Table 7 Entry 2

$3 P 705-1+C 6 D_{6}-C D_{3} \omega$

Archive directory: /export/home/auto/vnmrsys/data

Sample directory:
File: PROTON

Pulse Sequence: 52 pui

Solvent: $\mathrm{C606}$
Temp. $26.0 \mathrm{C}$
Mercury-4003

Re lax. de lay 1.000 sec

Acg. time 1.994 sec

Width $6410.3 \mathrm{~Hz}$

OBSERVE H1 $400.4698629 \mathrm{MHZ}$

T size 655356
Total time 0
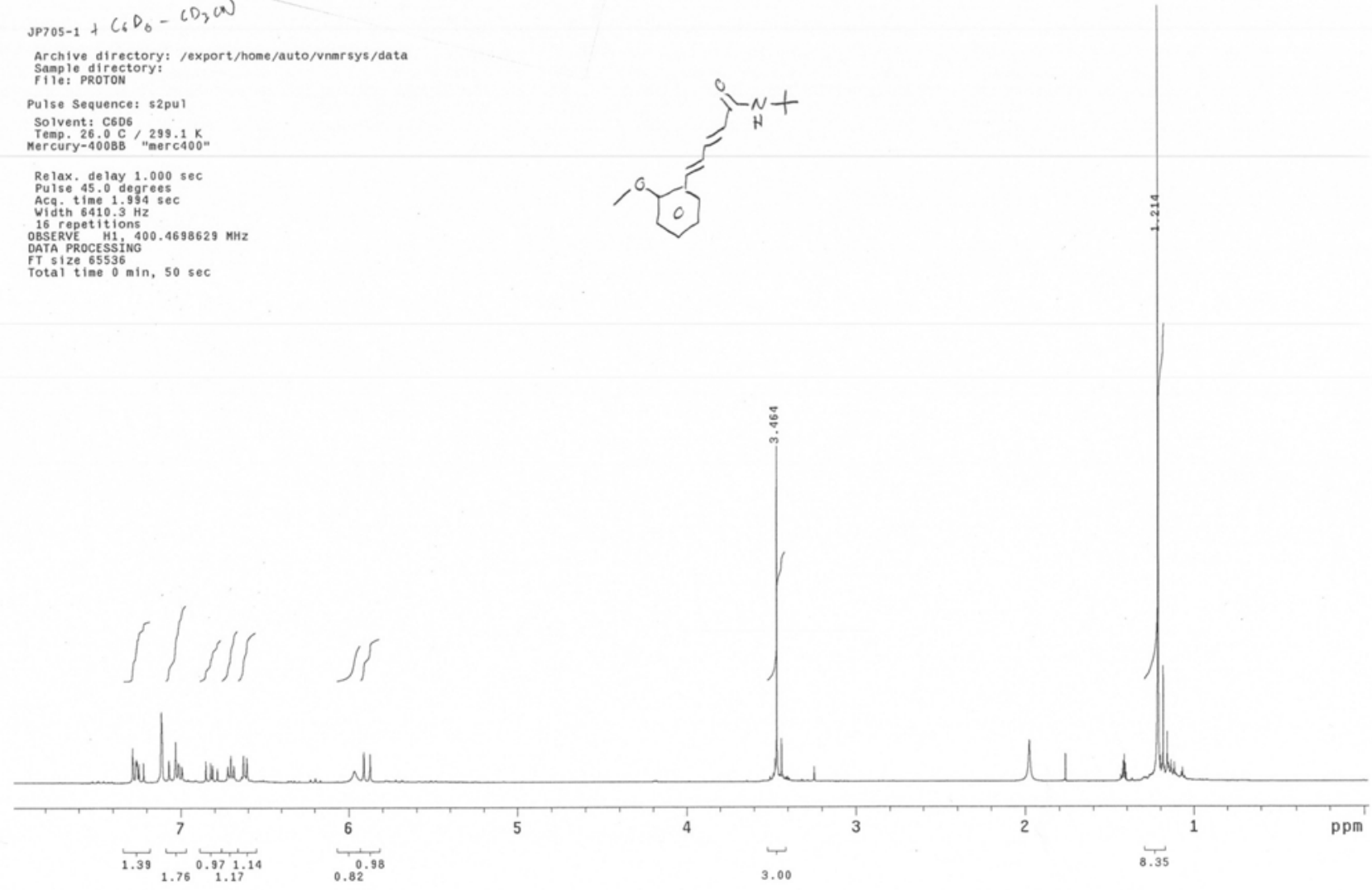

JP705-1

Archive directory: /export/home/auto/vnmrsys/data

Sample direct
FIle: CARBON

Pulse Sequence: s2pul

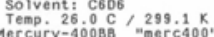

Relax. de lay $1.000 \mathrm{sec}$

Acq. time $1.199 \mathrm{sec}$

Width $25188.9 \mathrm{~Hz}$

OBSERE C13, 100.6981590 MHZ

Power 36 dB
continuous ly on

WALT $T$-15 modyulated

Line broadening $1.0 \mathrm{~Hz}$

Total time

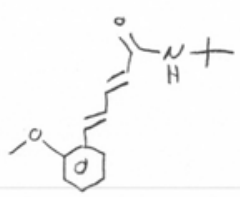

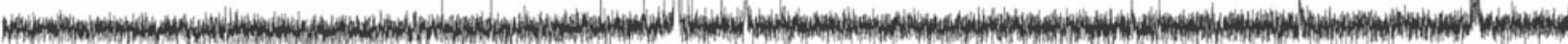




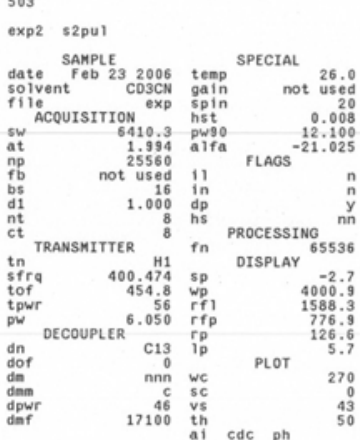

Table 7 Entry 3
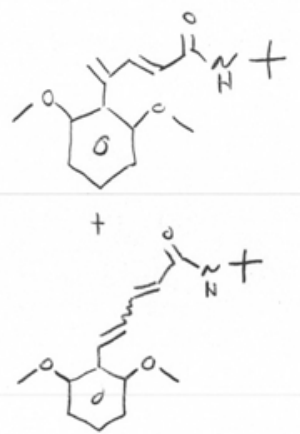
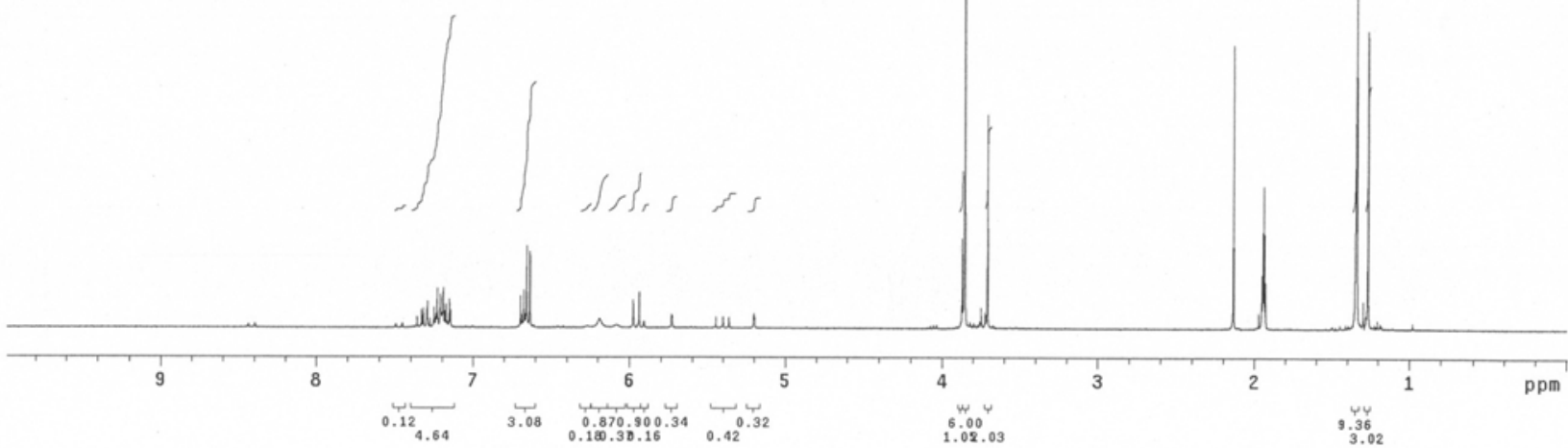

Table 7 Entry 4

492
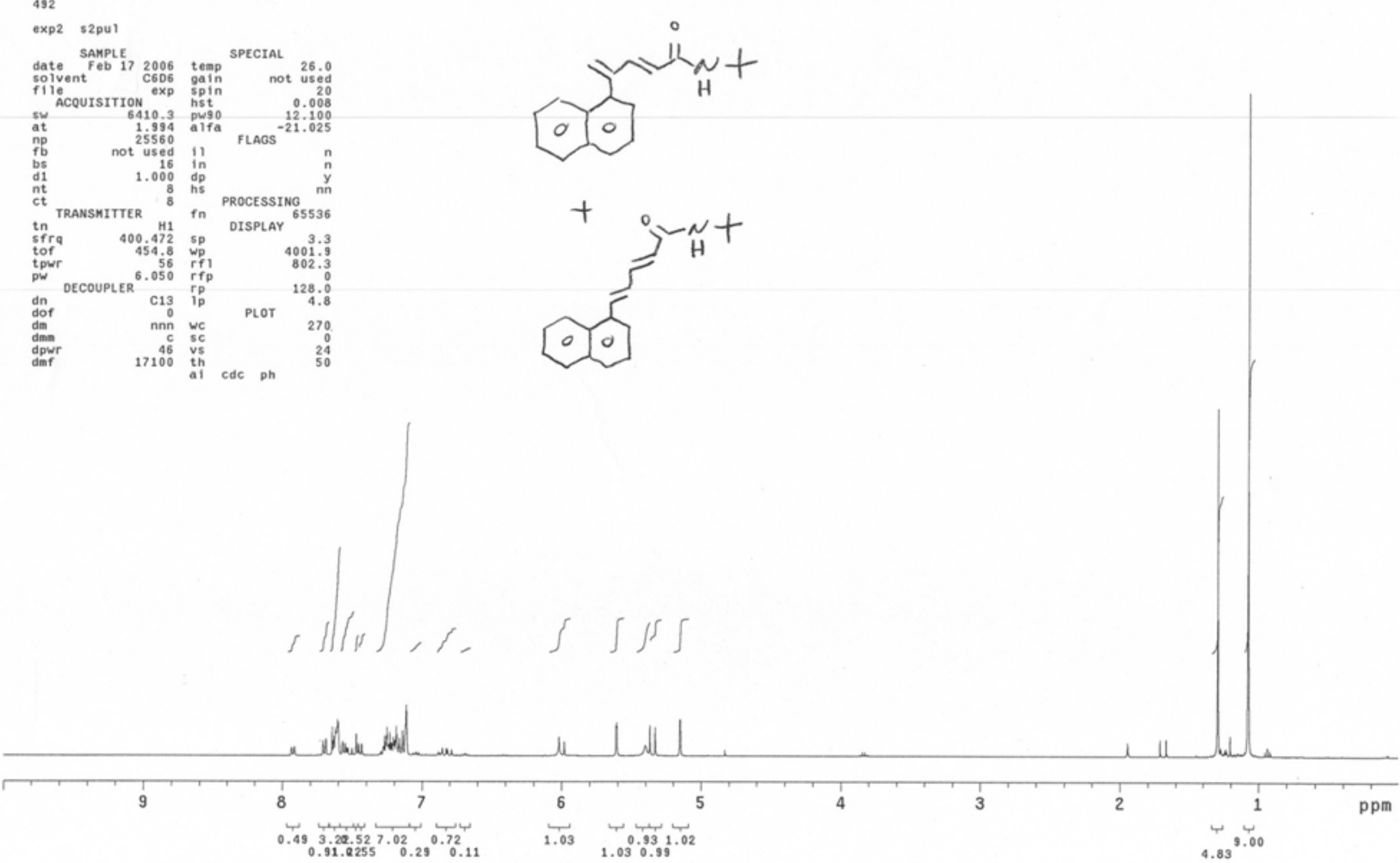
Table 7 Entry 5

$\begin{array}{ll}478 & \\ \exp 1 & 52 \mathrm{p} u 1\end{array}$
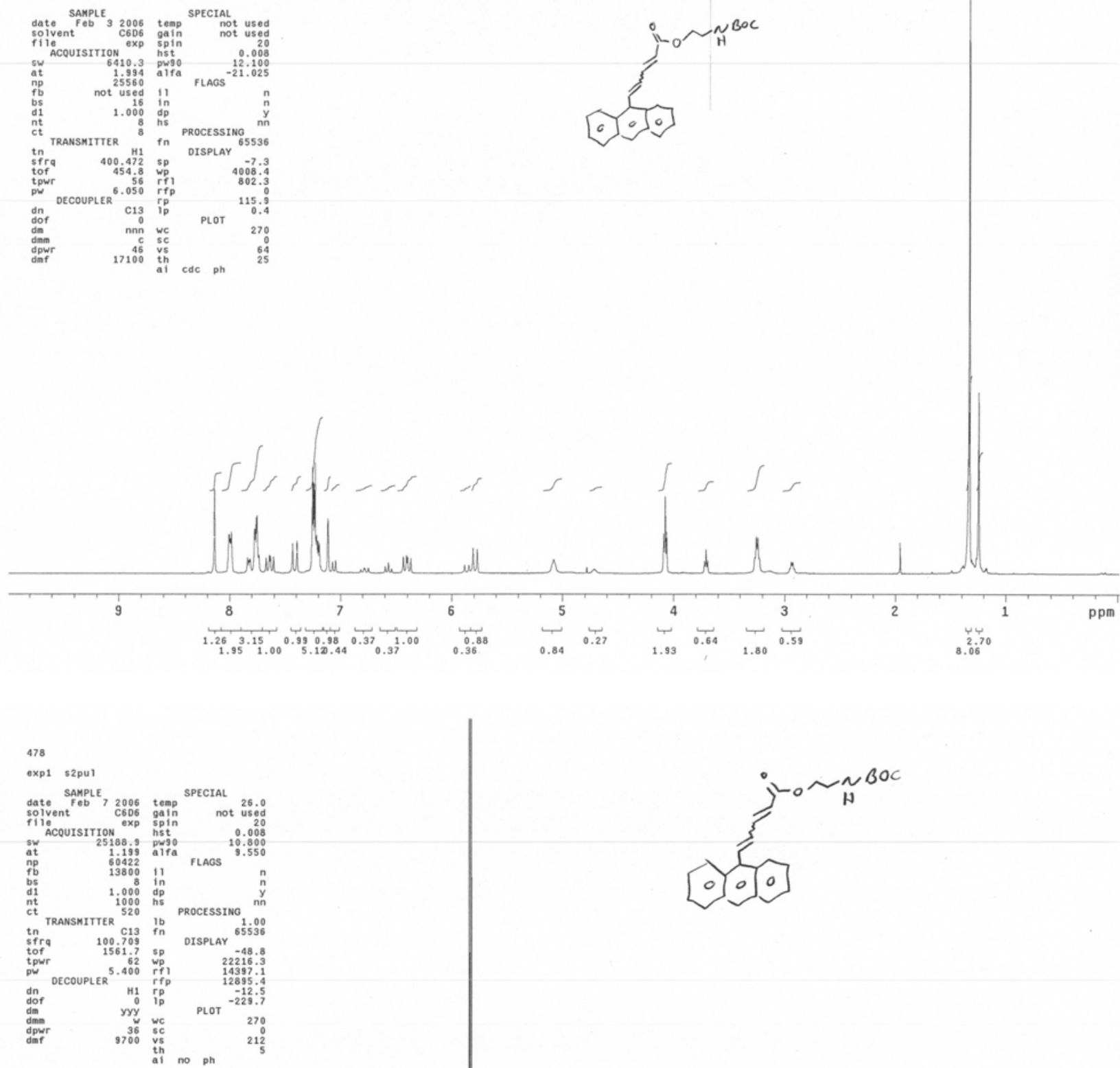

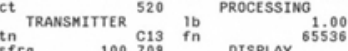

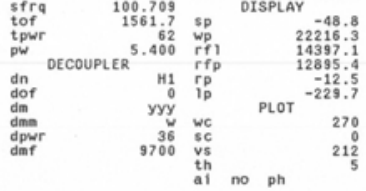

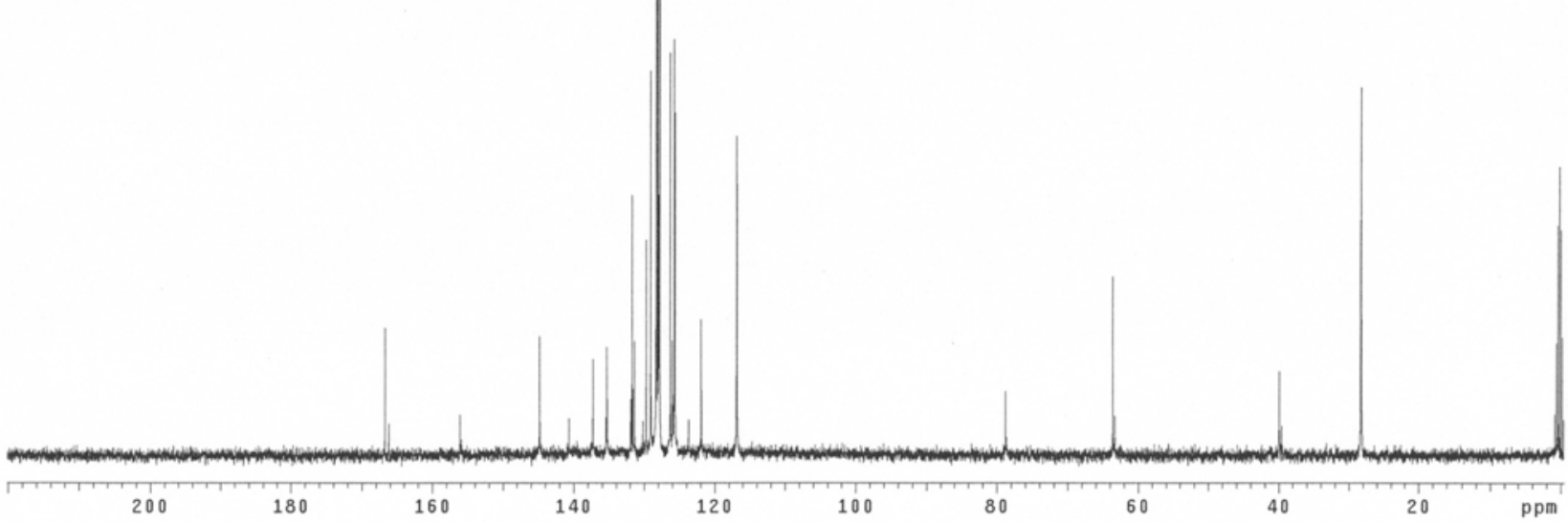


Table 7 Entry 6

766
$\exp 3 \quad 52 p u 1$

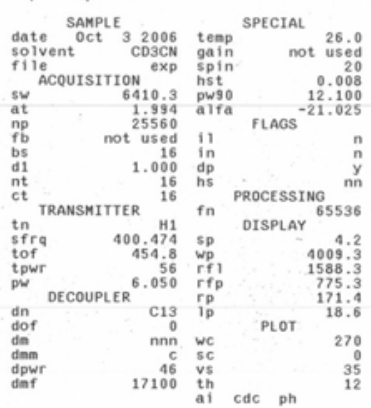<smiles>C=C(/C=C/C(=O)N=[V])C1C(F)C(F)C(F)C(F)C1F</smiles>

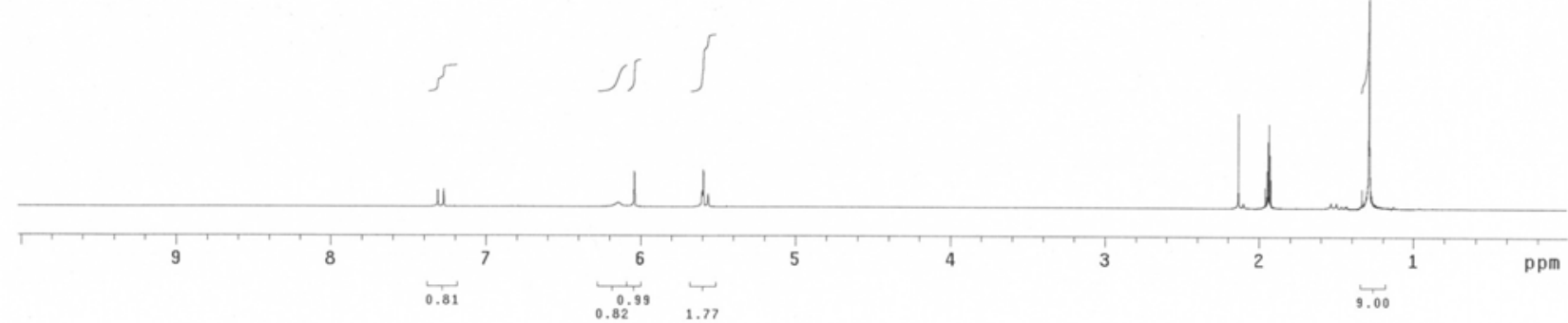

$\begin{array}{ll}766 & \\ \text { exp3 } & \text { s2pur }\end{array}$

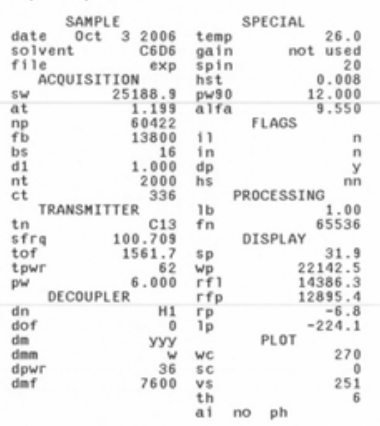

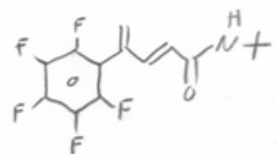

Only non-flourinated cambans 

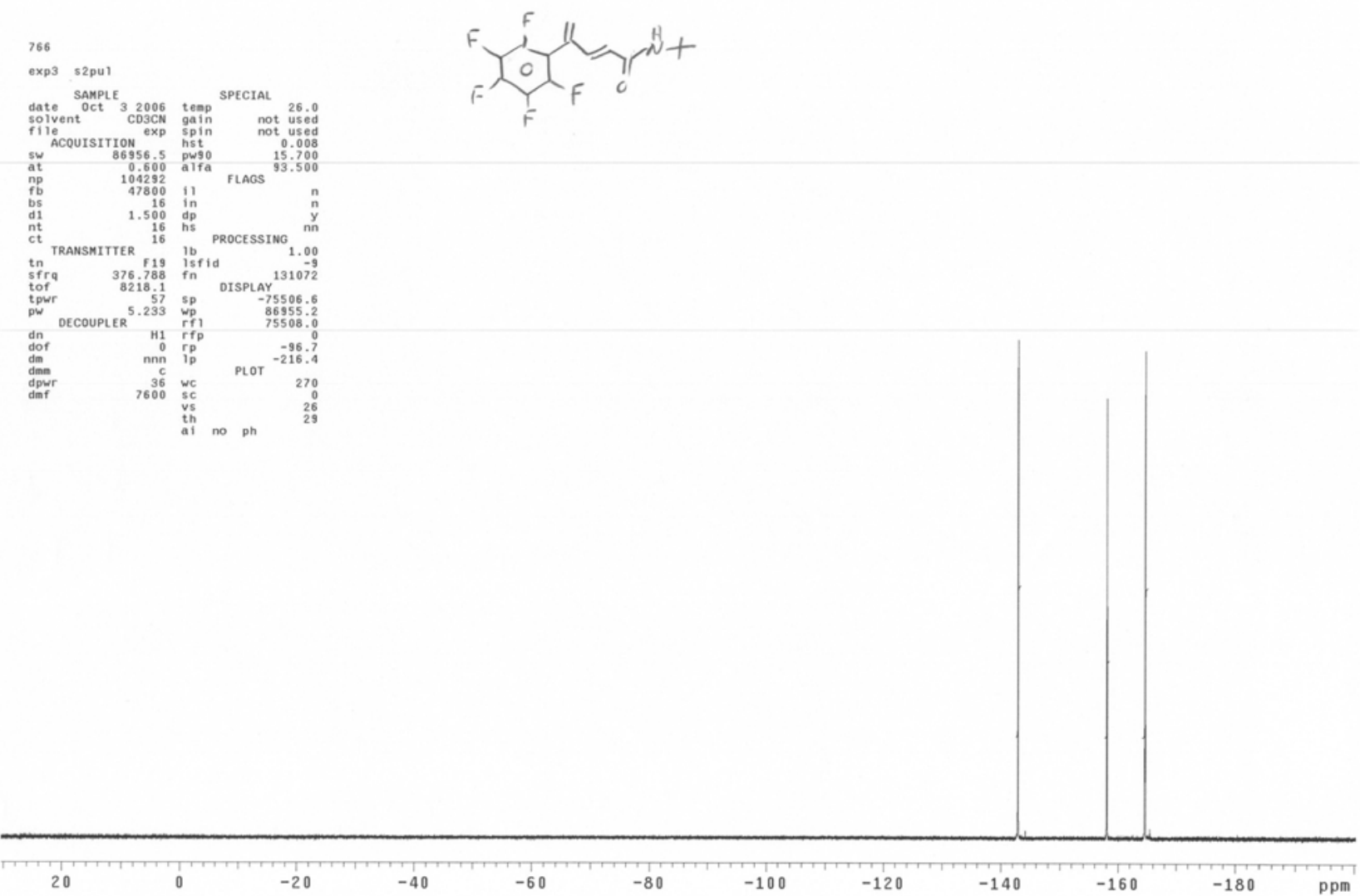
Compund 5

507

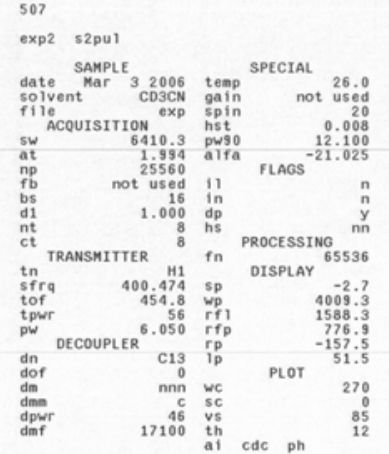

Compund 5

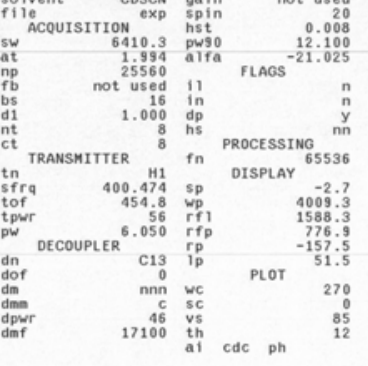
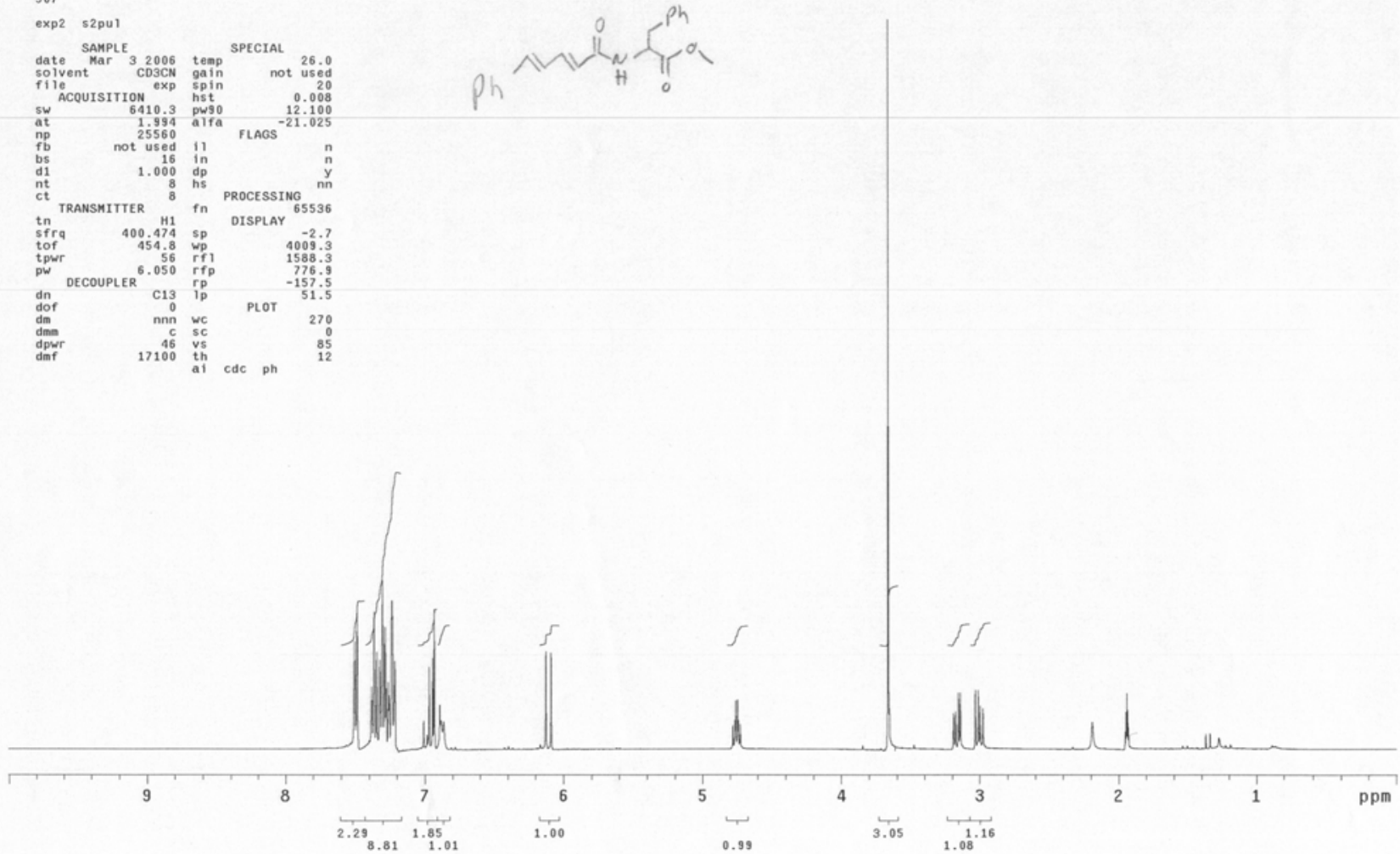

$\begin{array}{ll}507 & \\ \operatorname{exp2} & \text { 52pu1 }\end{array}$

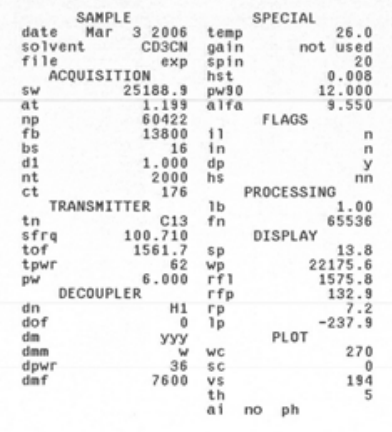
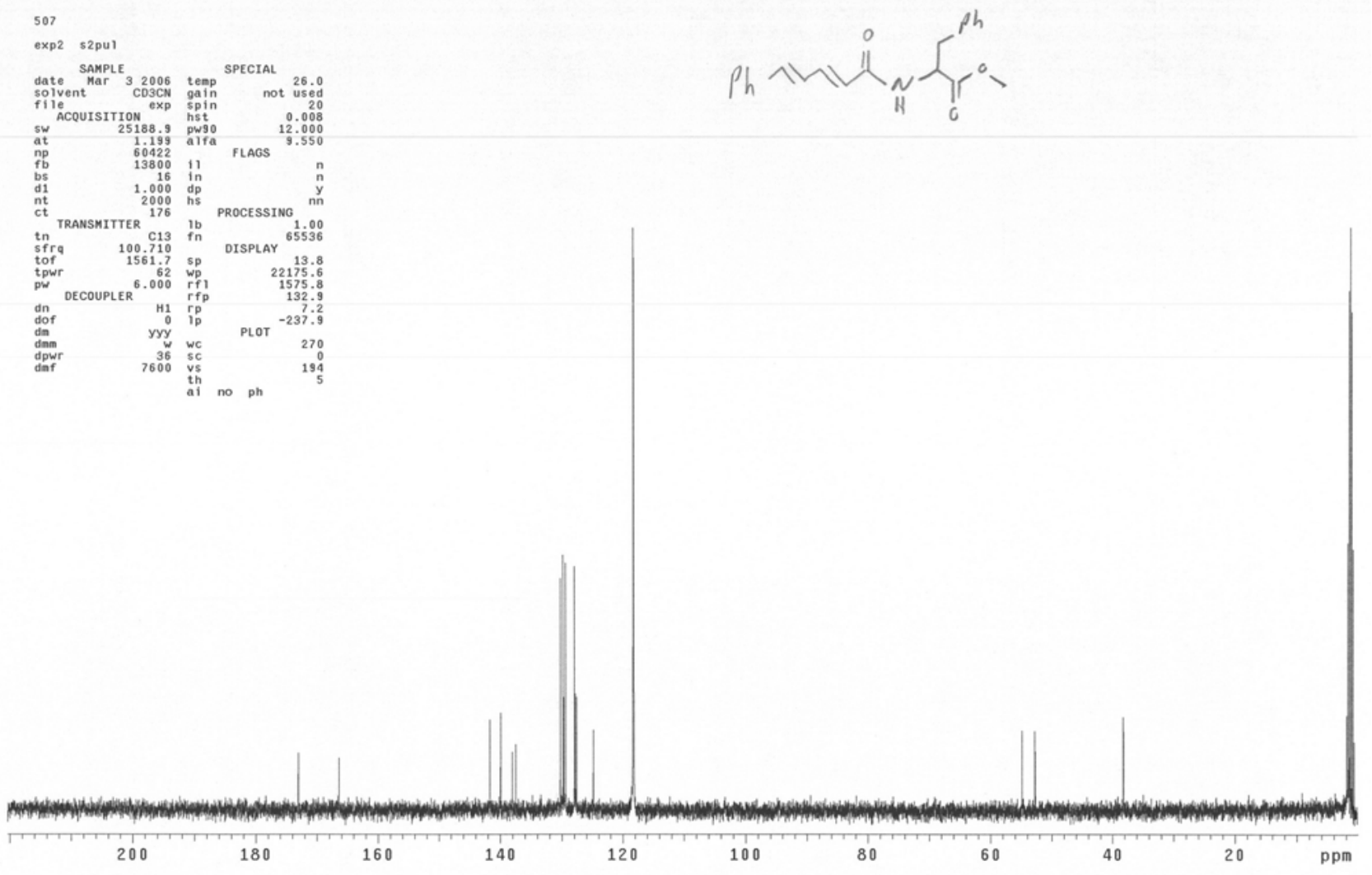